\title{
Asymptotic Stability of Traveling Wave Solutions of Systems for One-dimensional Gas Motion
}

\author{
Shuichi Kawashima ${ }^{1}$ and Akitaka Matsumura ${ }^{2}$ \\ 1 Department of Mathematics, Nara Women's University, Nara 630, Japan \\ 2 Department of Applied Mathematics and Physics, Kyoto University, Kyoto 606, Japan
}

\begin{abstract}
The asymptotic stability of traveling wave solutions with shock profile is investigated for several systems in gas dynamics. 1) The solution of a scalar conservation law with viscosity approaches the traveling wave solution at the rate $t^{-\gamma}$ (for some $\gamma>0$ ) as $t \rightarrow \infty$, provided that the initial disturbance is small and of integral zero, and in addition decays at an algebraic rate for $|x| \rightarrow \infty$. 2) The traveling wave solution with Nishida and Smoller's condition of the system of a viscous heat-conductive ideal gas is asymptotically stable, provided the initial disturbance is small and of integral zero. 3) The traveling wave solution with weak shock profile of the Broadwell model system of the Boltzmann equation is asymptotically stable, provided the initial disturbance is small and its hydrodynamical moments are of integral zero. Each proof is given by applying an elementary energy method to the integrated system of the conservation form of the original one. The property of integral zero of the initial disturbance plays a crucial role in this procedure.
\end{abstract}

\section{Contents}

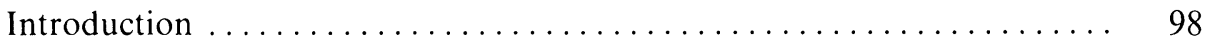

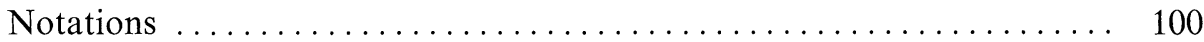

Section 1. Scalar conservation laws with viscosity

1.1 Traveling waves and main theorem.............. 101

1.2 Reformulation of the problem $\ldots \ldots \ldots \ldots \ldots \ldots \ldots \ldots \ldots$

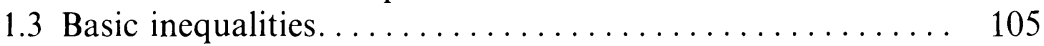

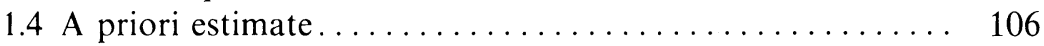

Section 2. Systems of viscous polytropic gases

2.1 Traveling waves and main theorem............. 108

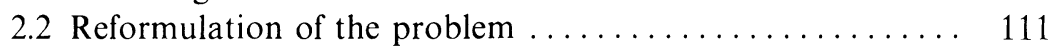

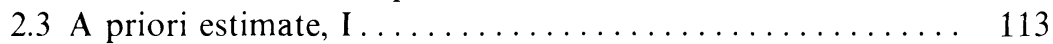

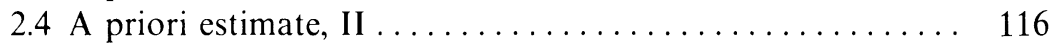


Section 3. The Broadwell model system

3.1 Traveling wave solution and main theorem.......... 118

3.2 Reformulation of the problem ................ 121

3.3 A priori estimate. . . . . . . . . . . . . . . . . . . 123

References

\section{Introduction}

The study of traveling wave solutions for systems of nonlinear partial differential equations is one of the interesting topics in mathematical physics. Recently new progress has been made in the study of the asymptotic stability of several interesting systems. Namely, Jones [5] proved the asymptotic stability of traveling wave solutions with a pulse profile for the FitzHugh-Nagumo system. His proof is based on the spectral analysis to the linearized system. Matsumura and Nishihara [6] showed the asymptotic stability of traveling wave solutions with shock profile for a model system of compressible viscous gas. Their result contains the strong shock case. They use an elemental energy method for the integrated system and this technique is based on the conservation form of the original system. Independently, using a similar energy method, Goodman [3] obtained a result concerning the asymptotic stability of traveling wave solutions with weak shock profile for systems of conservation laws with positive definite viscosity matrices.

In this paper, we shall apply these energy methods to the following equations (or systems) in gas dynamics and show the asymptotic stability of traveling wave solutions:

1) scalar conservation laws with viscosity,

2) systems of viscous heat-conductive ideal gases,

3) the Broadwell model system of the Boltzmann equation for a simple discrete velocity gas.

For scalar conservation laws with viscosity, Il'in and Oleinik [4] already proved the asymptotic stability of traveling wave solutions for initial disturbances with integral zero. They also showed that if the integral of the initial disturbance over $(-\infty, x]$ decays exponentially $e^{-\alpha|x|}$ (with some $\alpha>0$ ) for $|x| \rightarrow \infty$, then the solution approaches, in the maximum norm, the traveling wave solution at an exponential rate $e^{-\beta t}$ (for some $\beta>0$ ) as $t \rightarrow \infty$. The maximum principle plays an essential role in their proofs and so it is not straightforward to generalize these results to systems.

Recently, using an explicit formula of solutions, Nishihara [8] obtained a precise estimate of solutions to the Burgers equation. This estimate shows that if the integral of the initial disturbance over $(-\infty, x]$ has an algebraic order $O\left(|x|^{-\alpha}\right)$ (with some $\alpha>0$ ) for $|x| \rightarrow \infty$, then the solution converges, in the maximum norm, to the traveling wave solution at the same algebraic rate $t^{-\alpha}$ as $t \rightarrow \infty$. He also notes that this time decay rate is optimal in general.

In Sect. 1 we generalize Nishihara's time decay result to a class of scalar conservation laws with viscosity. We assume that the initial disturbance has zero integral. We also assume that the integral $\Psi_{0}(x)$ of the initial disturbance over $(-\infty, x]$ is suitably small in $H^{2}$ and belongs to a weighted $L^{2}$ space $L_{\alpha}^{2}$ (with some $\alpha>0$ ), that is, $(1+|x|)^{\alpha / 2} \Psi_{0} \in L^{2}$. Then it is proved that the solution converges, in $H^{1}$ 
norm, to the traveling wave solution at the rate $t^{-\beta / 2}$ with $\beta=[\alpha]$ as $t \rightarrow \infty$. When $\alpha$ is an integer, the exponent $\beta / 2$ of the time decay rate is equal to the exponent $\alpha / 2$ of the spatial decay rate of the integral $\Psi_{0}(x)$. Comparing this with Nishihara's result, we conjecture that the time decay rate obtained is optimal in our $L^{2}$ situations. To prove the above time decay result, we consider weighted $L^{2}$ spaces and use an energy method for the integrated equation which is derived on the basis of the conservation form of the original equation. The energy method works for the integrated form but does not for the original equation. The reason is because the monotonicity of the traveling wave solution gives a dissipative mechanism for the integrated equation but it produces an acceleration effect for the original equation. (See, for example, the Introduction of Goodman's paper [3].) This dissipation combined with the viscosity is sufficient to prove the stability of traveling wave solutions.

In Sect. 2 we treat the system of viscous heat-conductive gases in Lagrangian coordinates. The system consists of three conservation laws for the specific volume $v$, the velocity $u$ and the total energy $e+u^{2} / 2$, where $e$ is the internal energy. The existence of traveling wave solutions for this system is well investigated by Gilbarg [2] in general situations including the case of an ideal polytropic gas, i.e., the case where the pressure $p$ and the internal energy $e$ are given by

$$
p=R \theta / v \quad \text { and } \quad e=R \theta /(\gamma-1)+\text { constant, }
$$

respectively. Here $\theta$ is the absolute temperature. The constants $R>0$ and $\gamma>1$ denote the gas constant and the adiabatic exponent, respectively.

In the isentropic case, the pressure is given by $p=C v^{-\gamma}$ with a positive constant $C$ and the above equations can be reduced to a system of two conservation laws for $v$ and $u$. For this system, the asymptotic stability of traveling wave solutions was first proved by Matsumura and Nishihara [6]. More precisely, they assume that the initial disturbances for the conserved quantities $v$ and $u$ have zero integrals. Moreover, they assume that $\gamma-1$ times the shock strength of the traveling wave solution is suitably small and that the integrals of the initial disturbances over $(-\infty, x]$ are small in $H^{2}$. Under these conditions they proved that the solution converges, in the maximum norm, to the traveling wave solution as $t \rightarrow \infty$. The above condition for the traveling wave solution is the same type as those in Nishida and Smoller's paper [7] for the non-viscous case and in [10] for the viscous case. It should be noted that this condition does not exclude the case of strong shock when the adiabatic exponent $\gamma$ is close to 1 .

In Sect. 2 we generalize Matsumura and Nishihara's result for the system of an isentropic gas to the full system of an ideal polytropic gas. The result and the method are much the same as in [6]. We use an energy method for the integrated system. The advantage of the integrated form is even the same as in the scalar equation. In this case, however, there is a difficulty that the system considered is not parabolic, i.e., the viscosity matrix for the system is not positive definite. In fact, the second and the third equations of the system can be regarded as parabolic equations for the velocity $u$ and the absolute temperature $\theta$, respectively, while the first equation for the specific volume $v$ is not parabolic but hyperbolic. This difficulty of incomplete parabolicity can be overcome by using the technique which was developed in $[11,10]$ to prove the asymptotic stability of constant stationary solutions for the same system. There 
also appears a technical difficulty: while the conserved quantity for the third equation is not the absolute temperature but the total energy, the equation cannot be regarded as a parabolic equation for the total energy. Such a difficulty does not appear in the system of an isentropic gas considered in [6]. This difficulty will be overcome by approximating the perturbation of the absolute temperature by a new conserved quantity for which the integrated equation restores the parabolicity. Consequently, the stability analysis for the system considered here is much more complicated than those for the scalar parabolic equation and for the parabolic system considered in [3].

In the last section, we consider the Broadwel model system of the Boltzmann equation for a simple discrete velocity gas. This system consists of three equations. Only two of them can be reduced to conservation laws and hence the whole system is not of conservation form. The conserved quantities are called, as in the kinetic theory, the hydrodynamical moments; for the present system, they are the mass density and the momentum. The existence of traveling wave solutions for the system is well studied by Caflisch [1], though there have been no works on their asymptotic stability. In this paper, we apply an energy method similar to that in [6] or in Sect. 2 and show the asymptotic stability of traveling wave solutions. We assume that the initial disturbances for the hydrodynamical moments have zero integrals. We also assume that the shock strength of the traveling wave solution is suitably small. Moreover, suppose that the initial disturbance for the non-conserved quantity and the integrals of the initial disturbances for the hydrodynamical moments are small in $H^{1}$ and $H^{2}$, respectively. Then it is proved that the solution converges, in the maximum norm, to the traveling wave solution as $t \rightarrow \infty$. To prove this, we use the system which is derived from the original system by integrating the equations for the hydrodynamical moments. The energy method does work for this partially integrated system. But the system is purely hyperbolic, and the dissipation involved in the system seems to be weaker than that in the incompletely parabolic system considered in Sect. 2. So, the stability analysis is complicated.

\section{Notations}

We summarize the function spaces used in this paper. $L^{2}$ denotes the space of measurable functions on $\mathbb{R}$ which are square integrable, with the norm

$$
\|f\|=\left(\int|f(x)|^{2} d x\right)^{1 / 2} \text {. }
$$

$H^{l}(l \geqq 0)$ denotes the Sobolev space of $L^{2}$-functions $f$ on $\mathbb{R}$ whose derivatives $\partial_{x}^{j} f, j=1, \ldots, l$, are also $L^{2}$-functions, with norm

$$
\|f\|_{l}=\left(\sum_{j=0}^{l}\left\|\partial_{x}^{j} f\right\|^{2}\right)^{1 / 2} .
$$

$L_{\alpha}^{2}(\alpha \in \mathbb{R})$ denotes the space of measurable functions $f$ on $\mathbb{R}$ which satisfy $\langle x\rangle^{\alpha / 2} f \in L^{2}$, with the norm

$$
|f|_{\alpha}=\left(\int\langle x\rangle^{\alpha}|f(x)|^{2} d x\right)^{1 / 2},
$$

where $\langle x\rangle=\left(1+|x|^{2}\right)^{1 / 2}$. Note that $L^{2}=H^{0}=L_{0}^{2}$ and $\|\cdot\|=\|\cdot\|_{0}=|\cdot|_{0}$. 
Let $T$ and $B$ be a positive constant and a Banach space, respectively. $C^{k}(0, T ; B)(k \geqq 0)$ denotes the space of $B$-valued $k$-times continuously differentiable functions on $[0, T] . L^{2}(0, T ; B)$ denotes the space of $B$-valued $L^{2}$-functions on $[0, T]$. The corresponding spaces of $B$-valued functions on $[0, \infty)$ are denoted by $C^{k}(0, \infty ; B)$ and $L^{2}(0, \infty ; B)$, respectively.

\section{Scalar Conservation Laws with Viscosity}

1.1. Traveling Waves and Main Theorem. In this section we study a scalar conservation law of the form

$$
u_{t}+f(u)_{x}=\mu u_{x x}
$$

where $f$ is a smooth function on an interval $[\underline{u}, \bar{u}]$ such that

$$
f^{\prime \prime}(u)>0 \text { for all } u \in[\underline{u}, \bar{u}] \text {, }
$$

and $\mu$ is a positive constant.

The Eq. (1.1) admits smooth traveling wave solutions with shock profile

$$
\begin{aligned}
& u(t, x)=U(\xi), \quad \xi=x-s t \\
& U(\xi) \rightarrow u_{ \pm} \quad \text { as } \quad \xi \rightarrow \pm \infty,
\end{aligned}
$$

where $u_{+} \in(\underline{u}, \bar{u})$ and $s$ (the shock speed) are constants satisfying the RankineHugoniot condition

and the shock condition

$$
s\left(u_{+}-u_{-}\right)=f\left(u_{+}\right)-f\left(u_{-}\right),
$$

$$
f^{\prime}\left(u_{+}\right)<s<f^{\prime}\left(u_{-}\right)
$$

or, equivalently,

$$
u_{+}<u_{-} \text {. }
$$

In fact the function $U$ can be determined by the ordinary differential equation

$$
\mu U_{\xi}=-s U+f(U)+a \equiv-M(U),
$$

where $a=-s u_{ \pm}+f\left(u_{ \pm}\right)$is the integral constant. Note that the solution of (1.7) with condition (1.4) is unique up to a shift in $\xi$.

We consider the initial value problem for (1.1) with the initial condition

$$
u(0, x)=u_{0}(x)
$$

where $u_{0}$ is a bounded measurable function such that

$$
u_{0}(x) \rightarrow u_{ \pm} \quad \text { as } \quad x \rightarrow \pm \infty
$$

and the integrals

$$
\int_{-\infty}^{0}\left(u_{0}(x)-u_{-}\right) d x \text { and } \int_{0}^{+\infty}\left(u_{0}(x)-u_{+}\right) d x
$$

exist. Under these assumptions Il'in and Oleinik [4] proved that as $t \rightarrow \infty$, the 
solution $u(t, x)$ of the problem (1.1), (1.8) tends uniformly with respect to $x \in \mathbb{R}$ to the traveling wave solution $U(x-s t)$ which is uniquely determined by the relation

$$
\int_{-\infty}^{+\infty}\left(u_{0}-U\right)(x) d x=0
$$

Our aim is to show an algebraic decay rate $t^{-\gamma}(\gamma>0)$ under some additional assumptions. Let $U$ be a traveling wave solution. We assume that

$$
u_{0}-U \in H^{1}
$$

and the integral

$$
\Psi_{0}(x)=\int_{-\infty}^{x}\left(u_{0}-U\right)(y) d y
$$

exists for any $x \in \mathbb{R}$, and satisfies

$$
\Psi_{0} \in L_{\alpha}^{2} \text { for some } \alpha \geqq 0 .
$$

It should be noted that (1.11) and (1.13) imply (1.9) and (1.10).

Since $f$ in (1.1) is defined only on $[\underline{u}, \bar{u}]$, it is reasonable to assume

$$
u_{0}(x) \in[\underline{u}, \bar{u}] \text { for any } x \in \mathbb{R} .
$$

In the following we simply assume $\left\|u_{0}-U\right\|_{1} \leqq \varepsilon_{0}$ instead of the condition (1.14), where $\varepsilon_{0}$ is a positive constant such that

$$
\varepsilon_{0} \leqq \min \left\{u_{+}-\underline{u}, \quad \bar{u}-u_{-}\right\} .
$$

The main theorem in this section is the following:

Theorem 1.1. Let $u_{ \pm} \in(\underline{u}, \bar{u})$ and s satisfy (1.5) and (1.6), and let $U(x-s t)$ be a traveling wave solution which smoothly interpolates the asymptotic values $u_{ \pm}$with the speed $s$. Suppose that the initial data $u_{0}$ satisfy (1.11) and (1.13) for some $\alpha \geqq 0$. Then there exists a positive constant $\varepsilon_{1}\left(\leqq \varepsilon_{0}\right)$ such that if $\left\|u_{0}-U\right\|_{1}+\left\|\Psi_{0}\right\| \leqq \varepsilon_{1}$, the initial value problem (1.1), (1.8) has a unique global solution $u(t, x)$ with

$$
\left\{\begin{array}{l}
u-U \in C^{0}\left(0, \infty ; H^{1}\right) \cap L^{2}\left(0, \infty ; H^{2}\right) \\
u(t, x) \in[\underline{u}, \bar{u}] \text { for any } t \geqq 0, x \in \mathbb{R} .
\end{array}\right.
$$

Moreover, the solution tends in the maximum norm to the traveling wave solution at the rate $t^{-\gamma / 2}$ with $\gamma=[\alpha]$ :

$$
\sup _{x \in \mathbb{R}}|u(t, x)-U(x-s t)| \leqq C_{1}(1+t)^{-\gamma / 2}\left(\left\|u_{0}-U\right\|_{1}+\left|\Psi_{0}\right|_{\alpha}\right) \quad \text { for any } t \geqq 0,
$$

where $C_{1}$ is a positive constant.

For the proof, we need some properties of traveling wave solutions. Let $u_{*} \in\left(u_{+}, u_{-}\right)$be a unique state determined by

$$
s \equiv \frac{f\left(u_{+}\right)-f\left(u_{-}\right)}{u_{+}-u_{-}}=f^{\prime}\left(u_{*}\right),
$$

and let $U=U(\xi)$ be the traveling wave solution in Theorem 1.1. Since $M(u)=$ $s\left(u-u_{ \pm}\right)-\left(f(u)-f\left(u_{ \pm}\right)\right)>0$ for $u \in\left(u_{+}, u_{-}\right), U$ is strictly decreasing in $\xi \in \mathbb{R}$. 
Therefore there exists uniquely number $\xi_{*} \in \mathbb{R}$ such that

$$
U\left(\xi_{*}\right)=u_{*}
$$

The following result plays an important role in deriving estimates of weighted norm of solutions (see Lemma 1.6).

Lemma 1.2. For any $\beta \in[0, \alpha]$, there is a positive constant $c_{0}$ independent of $\beta$ such that

$$
A_{\beta}(\xi) \equiv \frac{1}{2}\left\{\beta\left(\xi-\xi_{*}\right)\left\langle\xi-\xi_{*}\right\rangle^{-1}\left(s-f^{\prime}(U)\right)-\left\langle\xi-\xi_{*}\right\rangle f^{\prime}(U)_{\xi}\right\} \geqq \beta c_{0}
$$

for any $\xi \in \mathbb{R}$, where $\langle\xi\rangle=\left(1+\xi^{2}\right)^{1 / 2}$.

Proof. From the inequality $U_{\xi}<0$ (for $\xi \in \mathbb{R}$ ) and the conditions (1.2), (1.18) and (1.6) we can deduce that $g(\xi) \equiv s-f^{\prime}(U(\xi))$ is an increasing function of $\xi \in \mathbb{R}$, and satisfies $g\left(\xi_{*}\right)=0, g^{\prime}\left(\xi_{*}\right)>0$, and $g(\xi) \rightarrow g_{+}>0$ (respectively $g_{-}<0$ ) as $\xi \rightarrow+\infty$ (respectively $-\infty)$, where $g^{\prime}\left(\xi_{*}\right)=\mu^{-1} f^{\prime \prime}\left(u_{*}\right) M\left(u_{*}\right)$ and $g_{ \pm}=s-f^{\prime}\left(u_{ \pm}\right)$. Therefore,

$$
\left(\xi-\xi_{*}\right)\left\langle\xi-\xi_{*}\right\rangle^{-1}\left(s-f^{\prime}(U)\right) \geqq \begin{cases}\frac{1}{2} g^{\prime}\left(\xi_{*}\right)\left(\xi-\xi_{*}\right)^{2} & \text { for } \xi \text { near } \xi_{*}, \\ c & \text { otherwise, }\end{cases}
$$

where $c$ is a positive constant. On the other hand, $-\left\langle\xi-\xi_{*}\right\rangle f^{\prime}(U)_{\xi}=$ $\left\langle\xi-\xi_{*}\right\rangle g^{\prime}(\xi)>0$ holds for $\xi \in \mathbb{R}$, and in particular, $-\left\langle\xi-\xi_{*}\right\rangle f^{\prime}(U)_{\xi} \geqq g^{\prime}\left(\xi_{*}\right) / 2$ for $\xi$ near $\xi_{*}$. These considerations prove the lemma.

1.2 Reformulation of the Problem. Letting $U(\xi)$ be the traveling wave solution in Theorem 1.1, we put

$$
U(t, x)=U(\xi)+\psi(t, \xi), \quad \xi=x-s t .
$$

Then the problem (1.1), (1.8) is reduced to

$$
\begin{gathered}
\psi_{t}-s \psi_{\xi}+\{f(U+\psi)-f(U)\}_{\xi}=\mu \psi_{\xi \xi}, \\
\psi(0, \xi)=\psi_{0}(\xi) \equiv\left(u_{0}-U\right)(\xi) .
\end{gathered}
$$

Inspired by the relation $\psi_{0}=\Psi_{0, \xi}$ (see (1.12)) we seek the solution of (1.21) in the form

$$
\psi=\Psi_{\xi}
$$

Substituting it into (1.21) and integrating once with respect to $\xi$, we get

$$
\Psi_{t}-s \Psi_{\xi}+f\left(U+\Psi_{\xi}\right)-f(U)=\mu \Psi_{\xi \xi},
$$

with the initial data

$$
\Psi(0, \xi)=\Psi_{0}(\xi)
$$

Let us define the solution space of (1.24) by

$$
X(0, T)=\left\{\bar{\Psi}_{\in} C^{0}\left(0, T ; H^{2}\right) ; \Psi_{\xi} \in L^{2}\left(0, T ; H^{2}\right)\right\}
$$

with $0<T \leqq+\infty$. Then the problem (1.24), (1.25) can be solved globaly in time as follows.

Theorem 1.3. Suppose $\Psi_{0} \in H^{2} \cap L_{\alpha}^{2}$ for some $\alpha \geqq 0$. Then there exist positive constants 
$\varepsilon_{2}\left(\leqq \varepsilon_{0}\right)$ and $C_{2}$ such that if $\left\|\Psi_{0}\right\|_{2} \leqq \varepsilon_{2}$, the problem (1.24),(1.25) has a unique global solution $\Psi \in X(0,+\infty)$ satisfying

$$
(1+t)^{\gamma}\|\Psi(t)\|_{2}^{2}+\int_{0}^{t}(1+\tau)^{\gamma}\left\|\Psi_{\xi}(\tau)\right\|_{2}^{2} d \tau \leqq C_{2}^{2}\left(\left|\Psi_{0}\right|_{\alpha}^{2}+\left\|\Psi_{0, \xi}\right\|_{1}^{2}\right)
$$

for $t \geqq 0$, where $0 \leqq \gamma \leqq[\alpha]$.

Here we note that Theorem 1.1 is a direct consequence of Theorem 1.3 because the solution of (1.1) is unique in the function space $C^{0}\left(0, T ; H^{1}\right) \cap L^{2}\left(0, T ; H^{2}\right)$. Therefore it is sufficient to prove Theorem 1.3 for our purpose. To do that, we shall combine a local existence result together with a priori estimates.

Proposition 1.4 (local existence). Suppose $\Psi_{0} \in H^{2}$ and $\left\|\Psi_{0}\right\|_{2} \leqq \varepsilon_{0} / 2$. Then there is a positive constant $T_{0}$ depending on $\varepsilon_{0}$ such that the problem (1.24), (1.25) has a unique solution $\Psi \in X\left(0, T_{0}\right)$ satisfying

$$
\|\Psi(t)\|_{2}^{2}+\int_{0}^{t}\left\|\Psi_{\xi}(\tau)\right\|_{2}^{2} d \tau \leqq 4\left\|\Psi_{0}\right\|_{2}^{2}
$$

for $t \in\left[0, T_{0}\right]$. Moreover, if $\Psi_{0} \in L_{\alpha}^{2}$ for some $\alpha \geqq 0$, then $\Psi \in C^{0}\left(0, T_{0} ; L_{\alpha}^{2}\right)$ and $\Psi_{\xi} \in L^{2}\left(0, T_{0} ; L_{\alpha}^{2}\right)$.

Proposition 1.5 (a priori estimate). Let $T$ be a positive constant. Suppose that the problem (1.24), (1.25) has a solution $\Psi \in X(0, T)$ satisfying $\Psi \in C^{0}\left(0, T ; L_{\alpha}^{2}\right)$ and $\Psi_{\xi} \in L^{2}\left(0, T ; L_{\alpha}^{2}\right)$ for some $\alpha \geqq 0$. Then for each $\beta \in[0, \alpha]$, there exist positive constants $\varepsilon_{3}\left(\leqq \varepsilon_{0}\right)$ and $C_{3}$, which are independent of $T$ and $\beta$, such that if $\sup \|\Psi(t)\|_{2} \leqq \varepsilon_{3}$, then the estimate $0 \leqq t \leqq T$

$$
(1+t)^{\gamma}\|\Psi(t)\|_{2}^{2}+\int_{0}^{t}(1+\tau)^{\gamma}\left\|\Psi_{\xi}(\tau)\right\|_{2}^{2} d \tau \leqq C_{3}^{2}\left(\left|\Psi_{0}\right|_{\beta}^{2}+\left\|\Psi_{0, \xi}\right\|_{1}^{2}\right)
$$

holds for $t \in[0, T]$, where $0 \leqq \gamma \leqq[\beta]$.

Proposition 1.4 can be proved in the standard way. So we omit its proof. Proposition 1.5 will be proved in the following two subsections. Here we show Theorem 1.3 by the continuation arguments based on Propositions 1.4 and 1.5.

Proof of Theorem 1.3. Choose $\varepsilon_{2}$ and $C_{2}$ such that $\varepsilon_{2}=\min \left\{\varepsilon_{3} / 2, \varepsilon_{3} / 2 C_{3}\right\}, C_{2}=$ $C_{3}$. Then the local solution of (1.24), (1.25) can be continued globally in time, provided the smallness condition $\left\|\Psi_{0}\right\|_{2} \leqq \varepsilon_{2}$ is satisfied. In fact we have $\left\|\Psi_{0}\right\|_{2} \leqq \varepsilon_{2} \leqq \varepsilon_{3} / 2$. Therefore, by Proposition 1.4 , there is a positive constant $T_{0}=$ $T_{0}\left(\varepsilon_{3}\right)$ such that a solution exists on $\left[0, T_{0}\right]$ and satisfies $\|\Psi(t)\|_{2} \leqq 2\left\|\Psi_{0}\right\|_{2} \leqq \varepsilon_{3}$ for $t \in\left[0, T_{0}\right]$. Hence we can apply Proposition 1.5 with $T=T_{0}$, and get the estimate (1.28) for $t \in\left[0, T_{0}\right]$. In particular, putting $\beta=0$, we have $\|\Psi(t)\|_{2} \leqq C_{3}\left\|\Psi_{0}\right\|$ for $t \in\left[0, T_{0}\right]$. Noting that $\left\|\Psi\left(T_{0}\right)\right\|_{2} \leqq C_{3} \varepsilon_{2} \leqq \varepsilon_{3} / 2$, we apply Proposition 1.4 by taking $t=T_{0}$ as the new initial time. Then we have a solution on $\left[T_{0}, 2 T_{0}\right]$ with the estimate $\|\Psi(t)\|_{2} \leqq 2\left\|\Psi\left(T_{0}\right)\right\|_{2} \leqq \varepsilon_{3}$ for $t \in\left[T_{0}, 2 T_{0}\right]$. Therefore $\|\Psi(t)\|_{2} \leqq \varepsilon_{3}$ holds on $\left[0,2 T_{0}\right]$. Hence Proposition 1.5 again gives the estimate (1.28) for $t \in\left[0,2 T_{0}\right]$. In the same way we can extend the solution to the interval $\left[0, n T_{0}\right]$ succesively, $n=1,2, \ldots$, and get a global solution. The estimate (1.26) is a consequence of $(1.28)$ with $\beta=\alpha$. This completes the proof of Theorem 1.3. 
1.3. Basic Inequalities. Let $\Psi \in X(0, T)$ (for some $T>0)$ be a solution of (1.24), (1.25) satisfying $\Psi \in C^{0}\left(0, T ; L_{\alpha}^{2}\right)$ and $\Psi_{\xi} \in L^{2}\left(0, T ; L_{\alpha}^{2}\right)$ for some $\alpha \geqq 0$. Put

$$
N(\mathrm{t})=\sup _{0 \leqq \tau \leqq t}\|\Psi(\tau)\|_{2} \quad \text { for } \quad t \in[0, T],
$$

and assume $N(T) \leqq \varepsilon_{0}$, where $\varepsilon_{0}$ is a constant in (1.15). In order to estimate the solution, we rewrite Eq. (1.24) in the form

$$
\Psi_{t}-\left(s-f^{\prime}(U)\right) \Psi_{\xi}-\mu \Psi_{\xi \xi}=F\left(U, \Psi_{\xi}\right)
$$

where

$$
F(U, \psi)=-\left\{f(U+\psi)-f(U)-f^{\prime}(U) \psi\right\} .
$$

Lemma 1.6. For any $\beta, \gamma \in[0, \alpha]$, there is a positive constant $C$ independent of $T, \beta$ and $\gamma$ such that

$$
\begin{aligned}
&(1+t)^{\gamma}|\Psi(t)|_{\beta}^{2}+\beta \int_{0}^{t}(1+\tau)^{\gamma}|\Psi(\tau)|_{\beta-1}^{2} d \tau+\int_{0}^{t}(1+\tau)^{\gamma}\left|\Psi_{\xi}(\tau)\right|_{\beta}^{2} d \tau \\
& \leqq C\left\{\left|\Psi_{0}\right|_{\beta}^{2}+\gamma \int_{0}^{t}(1+\tau)^{\gamma-1}|\Psi(\tau)|_{\beta}^{2} d \tau+\beta \int_{0}^{t}(1+\tau)^{\gamma}\left\|\Psi_{\xi}(\tau)\right\|^{2} d \tau\right. \\
&\left.+\int_{0}^{t} \int(1+\tau)^{\gamma}\langle\xi\rangle^{\beta}|\Psi|\left|F\left(U, \Psi_{\xi}\right)\right| d \xi d \tau\right\}
\end{aligned}
$$

holds for $t \in[0, T]$.

Proof. Let $\xi_{*}$ be the constant in (1.18). Multiplying (1.29) by $(1+t)^{\gamma}\left\langle\xi-\xi_{*}\right\rangle^{\beta} \Psi$, we have

$$
\begin{aligned}
\left\{\frac{1}{2}(1+t)^{\gamma}\left\langle\xi-\xi_{*}\right\rangle^{\beta} \Psi^{2}\right\}_{t}-\frac{\gamma}{2}(1+t)^{\gamma-1}\left\langle\xi-\xi_{*}\right\rangle^{\beta} \Psi^{2} \\
\quad+(1+t)^{\gamma}\left\langle\xi-\xi_{*}\right\rangle^{\beta-1} A_{\beta}(\xi) \Psi^{2}+\mu(1+t)^{\gamma}\left\langle\xi-\xi_{*}\right\rangle^{\beta} \Psi_{\xi}^{2} \\
\quad+\beta \mu(1+t)^{\gamma}\left\langle\xi-\xi_{*}\right\rangle^{\beta-2}\left(\xi-\xi_{*}\right) \Psi \Psi_{\xi}+\{\cdots\}_{\xi} \\
=(1+t)^{\gamma}\left\langle\xi-\xi_{*}\right\rangle^{\beta} \Psi \cdot F\left(U, \Psi_{\xi}\right),
\end{aligned}
$$

where $A_{\beta}(\xi)$ is defined in (1.19), and $\{\cdots\}_{\xi}$ denotes the term which disappears after integration with respect to $\xi \in \mathbb{R}$. Integrating (1.32) over $[0, t] \times \mathbb{R}$ and using the estimate (1.19), we have

$$
\begin{aligned}
&(1+t)^{\gamma}|\Psi(t)|_{\beta}^{2}+\beta \int_{0}^{t}(1+\tau)^{\gamma}|\Psi(\tau)|_{\beta-1}^{2} d \tau+\int_{0}^{t}(1+\tau)^{\gamma}\left|\Psi_{\xi}(\tau)\right|_{\beta}^{2} d \tau \\
& \leqq C\left\{\left|\Psi_{0}\right|_{\beta}^{2}+\gamma \int_{0}^{t}(1+\tau)^{\gamma-1}|\Psi(\tau)|_{\beta}^{2} d \tau+\beta \int_{0}^{t} \int(1+\tau)^{\gamma}\langle\xi\rangle^{\beta-1}\left|\Psi \Psi_{\xi}\right| d \xi d \tau\right. \\
&\left.+\int_{0}^{t} \int(1+\tau)^{\gamma}\langle\xi\rangle^{\beta}|\Psi|\left|F\left(U, \Psi_{\xi}\right)\right| d \xi d \tau\right\}
\end{aligned}
$$

with some constant $C$. To get the desired estimate (1.31), we must estimate the third term on the right-hand side of (1.33). Using Schwarz' inequality, we have

$$
\beta C \int\langle\xi\rangle^{\beta-1}\left|\Psi \Psi_{\xi}\right| d \xi \leqq \frac{\beta}{2}|\Psi|_{\beta-1}^{2}+\beta C \int\langle\xi\rangle^{\beta-1} \Psi_{\xi}^{2} d \xi
$$


with a constant $C$. We choose a constant $R$ so large that $\alpha C\langle\xi\rangle^{-1} \leqq 1 / 2$ for any $|\xi| \geqq R$, and divide the integral on the right-hand side into two parts $I_{1}$ and $I_{2}$ according to the regions $|\xi| \geqq R$ and $|\xi| \leqq R$. Then we have the estimates $I_{1} \leqq \frac{1}{2}\left|\Psi_{\xi}\right|_{\beta}^{2}$ and $I_{2} \leqq \beta C\|\Psi\|^{2}$ with some constant $C$. Substitution of these estimates into (1.33) yields (1.31). This completes the proof of Lemma 1.6.

For derivatives of the solution, we have the following estimates.

Lemma 1.7. Let $l=1$ and 2. For any $\gamma \in[0, \alpha]$, there is a positive constant $C$ independent of $T$ and $\gamma$ such that

$$
\begin{aligned}
&(1+t)^{\gamma}\left\|\partial_{\xi}^{l} \Psi(t)\right\|^{2}+\int_{0}^{t}(1+\tau)^{\gamma}\left\|\partial_{\xi}^{l+1} \Psi(\tau)\right\|^{2} d \tau \\
& \leqq C\left\{\left\|\partial_{\xi}^{l} \Psi_{0}\right\|^{2}+\int_{0}^{t}(1+\tau)^{\gamma}\left\|\Psi_{\xi}(\tau)\right\|_{l-1}^{2} d \tau\right. \\
&\left.+\int_{0}^{t} \int(1+\tau)^{\gamma}\left|\partial_{\xi}^{l-1} \Psi \| \partial_{\xi}^{l-1} F\left(U, \Psi_{\xi}\right)\right| d \xi d \tau\right\}
\end{aligned}
$$

holds for $t \in[0, T]$.

Proof. Let $l=1$ and 2. Apply $\partial_{\xi}^{l}$ to (1.29) and multiply it by $(1+t)^{\gamma} \partial_{\xi}^{l} \Psi$. Integrate the resulting equation over $[0, t] \times \mathbb{R}$. Then we can get the desired estimate $(1.34)_{l}$ in the same way as in the previous lemma. The details are omitted.

1.4. A Priori Estimate. We proceed to estimate the solution of the problem (1.24), (1.25). Put

$$
N_{\alpha}=\left|\Psi_{0}\right|_{\alpha}+\left\|\Psi_{0, \xi}\right\|_{1} \text { for } \alpha \geqq 0 .
$$

We first take $\beta=\gamma=0$ in the inequalities (1.31), (1.34) $)_{1}$ and $(1.34)_{2}$, and combine them successively. Then we have

$$
\begin{aligned}
\|\Psi(t)\|_{2}^{2}+\int_{0}^{t}\left\|\Psi_{\xi}(\tau)\right\|_{2}^{2} d \tau \leqq C & \left\{N_{0}^{2}+\int_{0}^{t} \int\left(|\Psi|+\left|\Psi_{\xi \xi}\right|\right)\left|F\left(U, \Psi_{\xi}\right)\right|\right. \\
& \left.+\left|\Psi_{\xi \xi \xi}\right|\left|F\left(U, \Psi_{\xi}\right)_{\xi}\right| d \xi d \tau\right\} .
\end{aligned}
$$

Since $F\left(U, \Psi_{\xi}\right)=0\left(\left|\Psi_{\xi}\right|^{2}\right)$ for $\left|\Psi_{\xi}\right| \rightarrow 0$ (see (1.30)), the integral on the right-hand side is majorized by

$$
C N(t) \int_{0}^{t}\left\|\Psi_{\xi}(\tau)\right\|_{2}^{2} d \tau
$$

with some constant $C=C\left(\varepsilon_{0}\right)$, where we have used $N(T) \leqq \varepsilon_{0}$. Therefore we arrive at the following lemma.

Lemma 1.8. There are positive constants $\varepsilon_{4}\left(\leqq \varepsilon_{0}\right)$ and $C=C\left(\varepsilon_{4}\right)$ independent of $T$ such that if $N(T) \leqq \varepsilon_{4}$, the estimate (1.28) with $\beta=\gamma=0$ holds for $t \in[0, T]$ :

$$
\|\Psi(t)\|_{2}^{2}+\int_{0}^{t}\left\|\Psi_{\xi}(\tau)\right\|_{2}^{2} d \tau \leqq C N_{0}^{2}
$$

Next, combining (1.31) with (1.35), we derive the decay estimate for $L^{2}$-norm of the solution. 
Lemma 1.9. Let $\gamma \in[0, \alpha] \cap Z$. There are positive constants $\varepsilon_{5}\left(\leqq \varepsilon_{4}\right)$ and $C=C\left(\varepsilon_{5}\right)$ independent of $T$ and $\gamma$ such that if $N(T) \leqq \varepsilon_{5}$, then

$$
(1+t)^{\gamma}|\Psi(t)|_{\alpha-\gamma}^{2}+(\alpha-\gamma) \int_{0}^{t}(1+\tau)^{\gamma}|\Psi(\tau)|_{\alpha-\gamma-1}^{2} d \tau+\int_{0}^{t}(1+\tau)^{\gamma}\left|\Psi_{\xi}(\tau)\right|_{\alpha-\gamma}^{2} d \tau \leqq C N_{\alpha}^{2}
$$

holds for $t \in[0, T]$. Consequently, for any $0 \leqq \gamma \leqq[\alpha]$, the following estimate holds:

$$
(1+t)^{\gamma}\|\Psi(t)\|^{2}+\int_{0}^{t}(1+\tau)^{\gamma}\left\|\boldsymbol{\Psi}_{\xi}(\tau)\right\|^{2} d \tau \leqq C N_{\alpha}^{2} .
$$

Proof. We first estimate the last integral on the right-hand side of (1.31). If $N(T) \leqq \varepsilon_{0}$, it is majorized by

$$
C N(t) \int_{0}^{t}(1+\tau)\left|\Psi_{\xi}(\tau)\right|_{\beta}^{2} d \tau
$$

with a constant $C=C\left(\varepsilon_{0}\right)$. Therefore, for suitably small $N(T)$, say $N(T) \leqq \varepsilon_{5}$, the inequality (1.31) becomes

$$
\begin{aligned}
& (1+t)^{\gamma}|\Psi(t)|_{\beta}^{2}+\beta \int_{0}^{t}(1+\tau)^{\gamma}|\Psi(\tau)|_{\beta-1}^{2} d \tau+\int_{0}^{t}(1+\tau)^{\gamma}\left|\Psi_{\xi}(\tau)\right|_{\beta}^{2} d \tau \\
& \quad \leqq C\left\{\left|\Psi_{0}\right|_{\beta}^{2}+\gamma \int_{0}^{t}(1+\tau)^{\gamma-1}|\Psi(\tau)|_{\beta}^{2} d \tau+\beta \int_{0}^{t}(1+\tau)^{\gamma}\left\|\Psi_{\xi}(\tau)\right\|^{2} d \tau\right\}
\end{aligned}
$$

with a constant $C=C\left(\varepsilon_{5}\right)$.

Step 1. Letting $\beta=\alpha$ and $\gamma=0$ in (1.38), we have (1.36) with $\gamma=0$, where (1.35) was used. Therefore the lemma is proved for $\alpha<1$.

Step 2. Firstly, letting $\beta=0$ and $\gamma=1$ in (1.38), and using (1.36) with $\gamma=0$, we have (1.37) with $\gamma=1$, where $\alpha \geqq 1$ is assumed. Secondly, letting $\beta=\alpha-1$ and $\gamma=1$ in (1.38), and using the estimates (1.36) with $\gamma=0$ and (1.37) with $\gamma=1$, we have the desired estimate (1.36) with $\gamma=1$. Therefore the proof is completed for $\alpha<2$.

Step 3. We repeat the same procedure as in Step 2. The estimate (1.38) (with $\beta=0$, $\gamma=2$ ) together with (1.36) (with $\gamma=1$ ) yields (1.37) (with $\gamma=2$ ), where $\alpha \geqq 2$ is assumed. Also, (1.38) (with $\beta=\alpha-2, \gamma=2$ ) together with (1.36) (with $\gamma=1$ ) and (1.37) (with $\gamma=2$ ) yields (1.36) (with $\gamma=2$ ), which proves the lemma for $\alpha<3$.

Repeating the same procedure, we can get the desired estimate (1.36) for any $\alpha \geqq 0$. This completes the proof of Lemma 1.9.

Finally, we show the same decay rate $t^{-\gamma / 2}$ for derivatives of the solution.

Lemma 1.10. Let $l=1$ and 2. For any $0 \leqq \gamma \leqq[\alpha]$, there are positive constants $\varepsilon_{6}\left(\leqq \varepsilon_{5}\right)$ and $C=C\left(\varepsilon_{6}\right)$ independent of $T$ and $\gamma$ such that if $N(T) \leqq \varepsilon_{6}$, then the decay estimate

$$
(1+t)^{\gamma}\left\|\partial_{\xi}^{l} \Psi(t)\right\|^{2}+\int_{0}^{t}(1+\tau)^{\gamma}\left\|\partial_{\xi}^{l+1} \Psi(\tau)\right\|^{2} d \tau \leqq C N_{\alpha}^{2}
$$

holds for $t \in[0, T]$.

Proof. We combine $(1.34)_{l}(l=1,2)$ and (1.37). If $N(T) \leqq \varepsilon_{0}$, the last integral on the 
right-hand side of $(1.34)_{1}$ is majorized by

$$
C N(t) \int_{0}^{t}(1+\tau)^{\gamma}\left\|\Psi_{\xi}(\tau)\right\|_{1}^{2} d \tau
$$

Therefore, for suitably small $N(T)$, we have

$$
(1+t)^{\gamma}\left\|\boldsymbol{\Psi}_{\xi}(t)\right\|^{2}+\int_{0}^{t}(1+\tau)^{\gamma}\left\|\Psi_{\xi \xi}(\tau)\right\|^{2} d \tau \leqq C\left\{\left\|\Psi_{0, \xi}\right\|^{2}+\int_{0}^{t}(1+\tau)^{\gamma}\left\|\Psi_{\xi}(\tau)\right\|^{2} d \tau\right\} .
$$

This inequality together with (1.37) gives the desired estimate (1.39) $)_{1}$. Similarly, we can obtain $(1.39)_{2}$ using the estimates $(1.34)_{2},(1.37)$ and $(1.39)_{1}$. This completes the proof of Lemma 1.10.

Now, the estimate $(1.28)$ follows directly from $(1.37),(1.39)_{1}$ and $(1.39)_{2}$. Therefore the proof of Proposition 1.5 is completed.

\section{Systems of Viscous Polytropic Gases}

2.1. Traveling Waves and Main Theorem. The one-dimensional motion of a viscous heat-conductive polytropic gas is described by the following system of equations in Lagrangian coordinates:

$$
\left\{\begin{array}{l}
v_{t}-u_{x}=0 \\
u_{t}+p_{x}=\left(\mu u_{x} / v\right)_{x} \\
\left(e+u^{2} / 2\right)_{t}+(p u)_{x}=\left(\kappa \theta_{x} / v+\mu u u_{x} / v\right)_{x}
\end{array}\right.
$$

where the unknowns $v>0, u$ and $\theta>0$ represent the specific volume, velocity and absolute temperature of the gas, respectively. The pressure $p$ and the internal energy $e$ are related with $v$ and $\theta$ by the equations of state

$$
p=R \theta / v, \quad e=R \theta /(\gamma-1)+\text { constant, }
$$

where $R>0$ is the gas constant and $\gamma \in(1,2]$ is the adiabatic exponent. The coefficient of viscosity and heat-conductivity, $\mu$ and $\kappa$, are assumed to be positive constants.

The system (2.1) admits smooth traveling wave solutions with shock profile

$$
\begin{array}{lll}
(v, u, \theta)(t, x)=(V, U, \Theta)(\xi), & & \xi=x-s t, \\
(V, U, \Theta)(\xi) \rightarrow\left(v_{ \pm}, u_{ \pm}, \theta_{ \pm}\right) & \text {as } & \xi \rightarrow \pm \infty,
\end{array}
$$

where $v_{ \pm}>0, u_{ \pm}, \theta_{ \pm}>0$ and $s$ are constants satisfying the Rankine-Hugoniot condition

$$
\left\{\begin{array}{l}
-s\left(v_{+}-v_{-}\right)-\left(u_{+}-u_{-}\right)=0 \\
-s\left(u_{+}-u_{-}\right)+\left(p_{+}-p_{-}\right)=0 \\
-s\left\{\left(e_{+}+u_{+}^{2} / 2\right)-\left(e_{-}+u_{-}^{2} / 2\right)\right\}+\left(p_{+} u_{+}-p_{-} u_{-}\right)=0
\end{array}\right.
$$

and Lax's shock condition that

$$
\text { either } \lambda_{1}^{+}<s<\lambda_{1}^{-} \text {or } \lambda_{3}^{+}<s<\lambda_{3}^{-} \text {, }
$$

where $\lambda_{1}=-(\gamma R \theta)^{1 / 2} / v, \lambda_{2}=0$ and $\lambda_{3}=(\gamma R \theta)^{1 / 2} / v$ are characteristic roots of the 
hyperbolic system associated with (2.1) (i.e., the system (2.1) with $\mu=\kappa=0$ ). In (2.5) and (2.6) we use the abbreviations $p_{+}=R \theta_{ \pm} / v_{+}, e_{ \pm}=R \theta_{ \pm} /(\gamma-1)+$ constant, $\lambda_{1}^{+}=$ $-\left(\gamma R \theta_{ \pm}\right)^{1 / 2} / v_{ \pm}$and $\lambda_{3}^{ \pm}=\left(\gamma R \theta_{ \pm}\right)^{1 / 2} v_{ \pm}$. Note that $(2.6)$ is equivalent to

$$
u_{+}<u_{-} \quad\left(\text { or } s\left(v_{+}-v_{-}\right)>0\right) \text {. }
$$

The functions $(V, U, \Theta)$ are determined by

$$
\left\{\begin{array}{l}
-s V-U=a_{1}, \\
-s U+P=\mu U_{\xi} / V+a_{2}, \\
-s\left(E+U^{2} / 2\right)+P U=\kappa \Theta_{\xi} / V+\mu U U_{\xi} / V+a_{3}
\end{array}\right.
$$

where $P=R \Theta / V$ and $E=R \Theta /(\gamma-1)+$ constant, and $a_{1}=-\left(s v_{ \pm}+u_{ \pm}\right), a_{2}=$ $-s u_{ \pm}+p_{ \pm}$and $a_{3}=-s\left(e_{ \pm}+u_{ \pm}^{2} / 2\right)+p_{ \pm} u_{ \pm}$are the integral constants. The system (2.7) is transformed into ([2])

$$
\left\{\begin{array}{l}
s \mu V_{\xi} / V=-\left\{P+s^{2}\left(V-b_{1} / s^{2}\right)\right\} \\
\kappa \Theta_{\xi} / s V=-\left\{E-\left(s^{2} / 2\right)\left(V-b_{1} / s^{2}\right)^{2}+b_{1}^{2} / 2 s^{2}-b_{2}\right\} \\
U=-\left(s V+a_{1}\right)
\end{array}\right.
$$

where $b_{1}=-s a_{1}+a_{2}=p_{ \pm}+s^{2} v_{ \pm}$and $b_{2}=\left(s a_{1}^{2} / 2-a_{1} a_{2}-a_{3}\right) / s=e_{ \pm}+p_{ \pm} v_{ \pm}+$ $s^{2} v_{ \pm}^{2} / 2$. See [2] for the existence and uniqueness (up to a shift in $\xi$ ) of the solution of (2.8) with the condition (2.4).

We consider the initial value problem for (2.1) in a neighborhood of a traveling wave solution, with the initial data

$$
(v, u, \theta)(0, x)=\left(v_{0}, u_{0}, \theta_{0}\right)(x) .
$$

Let $(V, U, \Theta)$ be a traveling wave solution with shock profile. We asume, as in Sect. 2, that

$$
\left(v_{0}-V, u_{0}-U, \theta_{0}-\Theta\right) \in H^{1}
$$

and the integrals

$$
\begin{aligned}
& \Phi_{0}(x)=\int_{-\infty}^{x}\left(v_{0}-V\right)(y) d y, \quad \Psi_{0}(x)=\int_{-\infty}^{x}\left(u_{0}-U\right)(y) d y, \\
& \tilde{W}_{0}(x)=\int_{-\infty}^{x}\left(e_{0}+u_{0}^{2} / 2\right)(y)-\left(E+U^{2} / 2\right)(y) d y
\end{aligned}
$$

exist for any $x \in \mathbb{R}$, and satisfy

$$
\left(\Phi_{0}, \Psi_{0}, W_{0}\right) \in L^{2}
$$

where $W_{0}=(\gamma-1)\left(\tilde{W}_{0}-U \Psi_{0}\right) / R$. In the above we put $e_{0}=R \theta_{0} /(\gamma-1)+$ constant. Here we note that $(2.10)$ and (2.12) imply the relations

$$
\left\{\begin{array}{l}
\int_{-\infty}^{+\infty}\left(v_{0}-V\right)(x) d x=0, \quad \int_{-\infty}^{+\infty}\left(u_{0}-U\right)(x) d x=0, \\
\int_{-\infty}^{+\infty}\left(e_{0}+u_{0}^{2} / 2\right)(x)-\left(E+U^{2} / 2\right)(x) d x=0 .
\end{array}\right.
$$

Let $\underline{v}<\bar{v}, \bar{u}$ and $\underline{\theta}<\bar{\theta}$ be any fixed positive constants. Suppose that the constants 
$v_{ \pm}, u_{ \pm}$and $\theta_{ \pm}$in (2.5) and (2.6) satisfy the following inequalities uniformly with respect to $\gamma \in(1,2]$.

$$
\underline{v} \leqq v_{ \pm} \leqq \bar{v}, \quad\left|u_{ \pm}\right| \leqq \bar{u}, \quad \underline{\theta} \leqq \theta_{ \pm} \leqq \bar{\theta} .
$$

As in Sect. 1, we choose a positive constant $\varepsilon_{0}$ such that

$$
\varepsilon_{0}<\min \{\underline{v}, \underline{\theta}\},
$$

and assume $\left\|v_{0}-V, \theta_{0}-\Theta\right\|_{1} \leqq \varepsilon_{0}$ instead of the condition $\inf \left\{v_{0}(x), \theta_{0}(x)\right.$; $x \in \mathbb{R}\}>0$.

The initial value problem (2.1), (2.9) is solved globally in time as follows.

Theorem 2.1. Let $v_{ \pm}, u_{ \pm}, \theta_{ \pm}$and $s$ be given constants satisfying (2.5), (2.6) and (2.14), and let $(V, U, \Theta)(\xi), \xi=x-s t$, be a traveling wave solution which smoothly interpolates the asymptotic values $\left(v_{+}, u_{+}, \theta_{+}\right)$with speed $s$. Suppose that the initial data $\left(v_{0}, u_{0}, \theta_{0}\right)$ satisfy (2.10) and (2.12). Put

$$
N_{0}=\left\|v_{0}-V, u_{0}-U,\left(\theta_{0}-\Theta\right) /(\gamma-1)^{1 / 2}\right\|_{1}+\left\|\Phi_{0}, \Psi_{0}, W_{0} /(\gamma-1)^{1 / 2}\right\| .
$$

Then there exist positive constants $\delta_{1}$ and $\varepsilon_{1}\left(\leqq \varepsilon_{0}\right)$, which are independent of $\gamma \in(1,2]$ and $\left(v_{ \pm}, u_{ \pm}, \theta_{ \pm}\right)$, such that if $(\gamma-1)\left|v_{+}-v_{-}\right| \leqq \delta_{1}$ and $N_{0} \leqq \varepsilon_{1}$, then the initial value problem (2.1), (2.9) has a unique global solution $(v, u, \theta)(t, x)$ with

$$
\left\{\begin{array}{l}
v-V \in C^{0}\left(0, \infty ; H^{1}\right) \cap L^{2}\left(0, \infty ; H^{1}\right), \\
(u-U, \theta-\Theta) \in C^{0}\left(0, \infty ; H^{1}\right) \cap L^{2}\left(0, \infty ; H^{2}\right), \\
\inf \{v(t, x), \theta(t, x) ; t \geqq 0, x \in \mathbb{R}\}>0,
\end{array}\right.
$$

Moreover, the solution tends to the traveling wave solution in the maximum norm:

$$
\sup _{x \in \mathbb{R}}|(v, u, \theta)(t, x)-(V, U, \Theta)(x-s t)| \rightarrow 0 \quad \text { as } \quad t \rightarrow \infty .
$$

For the proof, we need some properties of traveling wave solutions. We first note that the Rankine-Hugoniot condition (2.5) gives

$$
s^{2}=\frac{\gamma R \theta_{-}}{v_{+} v_{-}}\left(1-\delta_{+}\right), \quad \theta_{+}=\theta_{-}\left(1-\frac{v_{+}+v_{-}}{v_{-}} \delta_{+}\right)
$$

for $v_{+}-v_{-}>0$, and

$$
s^{2}=\frac{\gamma R \theta_{+}}{v_{+} v_{-}}\left(1-\delta_{-}\right), \quad \theta_{-}=\theta_{+}\left(1-\frac{v_{+}+v_{-}}{v_{+}} \delta_{-}\right)
$$

for $v_{+}-v_{-}<0$, where $\delta_{ \pm}=d_{ \pm} /\left(1+d_{ \pm}\right)$with $d_{ \pm}=(\gamma-1)\left|v_{+}-v_{-}\right| / 2 v_{ \pm}$.

Lemma 2.2. The traveling wave solution $(V, U, \Theta)(\xi)$ satisfies $s V_{\xi}=-U_{\xi}>0$ and $s \Theta_{\xi}<0$ for any $\xi \in \mathbb{R}$. Moreover there is a constant $C$ independent of $\gamma \in(1,2]$ and $\left(v_{ \pm}, u_{ \pm}, \theta_{ \pm}\right)$such that for $\xi \in \mathbb{R}$,

$$
\left\{\begin{array}{l}
\left|V_{\xi}\right|,\left|V_{\xi \xi}\right|,\left|\Theta_{\xi \xi}\right| \leqq C\left|v_{+}-v_{-}\right|, \\
\left|\Theta_{\xi}\right| \leqq C(\gamma-1)\left|v_{+}-v_{-}\right|, \quad\left|\Theta_{\xi} / V_{\xi}\right| \leqq C(\gamma-1) .
\end{array}\right.
$$


The estimates in (2.19) can be shown by direct calculations. We omit the proof.

\subsection{Reformulation of the Problem. Setting}

$$
(v, u, \theta)(t, x)=(V, U, \Theta)(\xi)+(\phi, \psi, w)(t, \xi), \quad \xi=x-s t
$$

we rewrite the problem (2.1), (2.9) in the form

$$
\left\{\begin{array}{l}
\phi_{t}-s \phi_{\xi}-\psi_{\xi}=0, \\
\psi_{t}-s \psi_{\xi}+R\left(\frac{\Theta+w}{v+\phi}-\frac{\Theta}{V}\right)_{\xi}=\left\{\frac{\mu}{V+\phi} \psi_{\xi}+\left(\frac{\mu}{V+\phi}-\frac{\mu}{V}\right) U_{\xi}\right\}_{\xi} \\
\left(\frac{R}{\gamma-1} w+\frac{1}{2} \psi^{2}+U \psi\right)_{t}-s\left(\frac{R}{\gamma-1} w+\frac{1}{2} \psi^{2}+U \psi\right)_{\xi}+R\left\{\frac{\Theta+w}{V+\phi} \psi\right. \\
\left.\quad+\left(\frac{\Theta+w}{V+\phi}-\frac{\Theta}{V}\right) U\right\}_{\xi}=\left\{\frac{\kappa}{V+\phi} w_{\xi}+\left(\frac{\kappa}{V+\phi}-\frac{\kappa}{V}\right) \Theta_{\xi}\right\}_{\xi} \\
\quad+\left\{\frac{\mu}{V+\phi}\left(\psi \psi_{\xi}+U \psi_{\xi}+U_{\xi} \psi\right)+\left(\frac{\mu}{V+\phi}-\frac{\mu}{V}\right) U U_{\xi}\right\}_{\xi}, \\
\quad(\phi, \psi, w)(0, \xi)=\left(\phi_{0}, \psi_{0}, w_{0}\right)(\xi) \equiv\left(v_{0}-V, u_{0}-U, \theta_{0}-\Theta\right)(\xi) .
\end{array}\right.
$$

It should be noted that the third equation of $(2.21)$ is reduced to

$$
\begin{aligned}
& \frac{R}{\gamma-1}\left(w_{t}-s w_{\xi}\right)+R \frac{\Theta+w}{V+\phi} \psi_{\xi}+R\left(\frac{\Theta+w}{V+\phi}-\frac{\Theta}{V}\right) U_{\xi} \\
& =\left\{\frac{\kappa}{V+\phi} w_{\xi}+\left(\frac{\kappa}{V+\phi}-\frac{\kappa}{V}\right) \Theta_{\xi}\right\}_{\xi}+\frac{\mu}{V+\phi}\left(\psi_{\xi}^{2}+2 U_{\xi} \psi_{\xi}\right)+\left(\frac{\mu}{V+\phi}-\frac{\mu}{V}\right) U_{\xi}^{2} .
\end{aligned}
$$

Equation (2.23) is not of conservation form but it is regarded as a parabolic equation in $w$.

Set $\quad \tilde{w}_{0} \equiv\left(e_{0}+u_{0}^{2} / 2\right)-\left(E+U^{2} / 2\right)=R w_{0} /(\gamma-1)+\psi_{0}^{2} / 2+U \psi_{0}$. Then, from (2.11), we have $\left(\phi_{0}, \psi_{0}, \tilde{w}_{0}\right)=\left(\Phi_{0, \xi} \Psi_{0, \xi} \tilde{W}_{0, \xi}\right)$. Taking it into account, we seek the solution of the problem (2.21), (2.22) in the form

$$
(\phi, \psi, \tilde{w})=\left(\Phi_{\xi}, \Psi_{\xi}, \tilde{W}_{\xi}\right)
$$

where $\tilde{w}=R w /(\gamma-1)+\psi^{2} / 2+U \psi$. Let us introduce

$$
W=\frac{\gamma-1}{R}(\tilde{W}-U \Psi)
$$

Using this $W$, we can write $w$ in the form

$$
w=W_{\xi}+\frac{\gamma-1}{R}\left(U_{\xi} \Psi-\frac{1}{2} \psi^{2}\right) \quad \text { with } \quad \psi=\Psi_{\xi} .
$$

Substituting (2.24) into (2.21) and integrating once with respect to $\xi$, we get the system for $(\Phi, \Psi, W)$ : 


$$
\left\{\begin{array}{l}
\Phi_{t}-s \Phi_{\xi}-\Psi_{\xi}=0, \\
\Psi_{t}-s \Psi_{\xi}+R\left(\frac{\Theta+w}{V+\Phi_{\xi}}-\frac{\Theta}{V}\right)=\frac{\mu}{V+\Phi_{\xi}} \Psi_{\xi \xi}+\left(\frac{\mu}{V+\Phi_{\xi}}-\frac{\mu}{V}\right) U_{\xi}, \\
\frac{R}{\gamma-1}\left(W_{t}-s W_{\xi}\right)-s U_{\xi} \Psi+R \frac{\Theta+w}{V+\Phi_{\xi}} \Psi_{\xi} \\
=\frac{\kappa}{V+\Phi_{\xi}} w_{\xi}+\left(\frac{\kappa}{V+\Phi_{\xi}}-\frac{\kappa}{V}\right) \Theta_{\xi}+\frac{\mu}{V+\Phi_{\xi}}\left(\Psi_{\xi} \Psi_{\xi \xi}+U_{\xi} \Psi_{\xi}\right),
\end{array}\right.
$$

with $w$ given by (2.26), where we have used the relation (2.25). The initial condition for $(2.27)$ is

$$
(\Phi, \Psi, W)(0, \xi)=\left(\Phi_{0}, \Psi_{0}, W_{0}\right)(\xi)
$$

Before introducing the solution space, we first rewrite the system (2.27) in the form

$$
\left\{\begin{array}{l}
\Phi_{t}-s \Phi_{\xi}-\Psi_{\xi}=0, \\
\Psi_{t}-s \Psi_{\xi}-\frac{1}{V}\left(b_{1}-s^{2} V\right) \Phi_{\xi}+\frac{R}{V} W_{\xi}-\frac{\mu}{V} \Psi_{\xi \xi}+\frac{\gamma-1}{V} U_{\xi} \Psi=F_{1}, \\
\frac{R}{\gamma-1}\left(W_{t}-s W_{\xi}\right)+\left(b_{1}-s^{2} V\right) \Psi_{\xi}-\frac{\kappa}{V}\left(W_{\xi}+\frac{\gamma-1}{R} U_{\xi} \Psi\right)_{\xi}-s U_{\xi} \Psi \\
\quad+\frac{\kappa}{V^{2}} \Theta_{\xi} \Phi_{\xi}=F_{2},
\end{array}\right.
$$

where $F_{1}$ and $F_{2}$ are nonlinear terms with respect to $(\Phi, \Psi, W)$; they are expressed in terms of $(\phi, \psi, w)$ only, that is,

$$
\left\{\begin{aligned}
F_{1}= & \frac{\gamma-1}{2 V} \psi^{2}-\frac{\phi}{V(V+\phi)}\left\{\left(b_{1}-s^{2} V\right) \phi-R w+\mu \psi_{\xi}\right\} \\
F_{2}= & -\frac{\kappa(\gamma-1)}{R V} \psi \psi_{\xi}+\frac{\psi}{V+\phi}\left\{\left(b_{1}-s^{2} V\right) \phi-R w+\mu \psi_{\xi}\right\} \\
& -\frac{\kappa \phi}{V(V-\phi)}\left(w_{\xi}-\frac{1}{V} \Theta_{\xi} \phi\right)
\end{aligned}\right.
$$

In the above derivation, we have used the equality $\left(R \Theta-\mu U_{\xi}\right) / V=b_{1}-s^{2} V($ see (2.8)). From (2.29) and (2.30) the equations of $\Psi$ and $W$ can be regarded as a parabolic system, provided $|\phi, \psi|$ is small enough.

From these considerations we can define the solution space of the problem (2.27), (2.28), as follows.

$$
X(0, T)=\left\{(\Phi, \Psi, W) \in C^{0}\left(0, T ; H^{2}\right) ; \Phi_{\xi} \in L^{2}\left(0, T ; H^{1}\right),\left(\Psi_{\xi}, W_{\xi}\right) \in L^{2}\left(0, T ; H^{2}\right)\right\} .
$$

Then we have the following

Theorem 2.3. Suppose $\left(\Phi_{0}, \Psi_{0}, W_{0}\right) \in H^{2}$. Then there exist positive constant $\delta_{2}$, $\varepsilon_{2}\left(\leqq \varepsilon_{0}\right)$ and $C_{2}$ independent of $\gamma \in(1,2]$ and $\left(v_{ \pm}, u_{ \pm}, \theta_{ \pm}\right)$such that if $(\gamma-1)\left|v_{+}-v_{-}\right| \leqq \delta_{2}$ and 


$$
N_{0}=\left\|\Phi_{0}, \Psi_{0}, W_{0} /(\gamma-1)^{1 / 2}\right\|+\left\|\phi_{0}, \psi_{0}, w_{0} /(\gamma-1)^{1 / 2}\right\|_{1} \leqq \varepsilon_{2},
$$

then the problem (2.27), (2.28) has a unique global solution $(\Phi, \Psi, W) \in X(0,+\infty)$ satisfying

$$
\begin{aligned}
& \left\|\left(\Phi, \Psi, W /(\gamma-1)^{1 / 2}\right)(t)\right\|^{2}+\left\|\left(\phi, \psi, w /(\gamma-1)^{1 / 2}\right)(t)\right\|_{1}^{2} \\
& \quad+\int_{0}^{t}\left\|\left|V_{\xi}\right|^{1 / 2}\left(\Psi, W /(\gamma-1)^{1 / 2}\right)(\tau)\right\|^{2}+\|\phi(\tau)\|_{1}^{2}+\|(\psi, w)(\tau)\|_{2}^{2} d \tau \leqq C_{2}^{2} N_{0}^{2}
\end{aligned}
$$

for $t \in[0, \infty)$, where $(\phi, \psi, w)$ is defined in (2.24) and (2.26), and $\left(\phi_{0}, \psi_{0}, w_{0}\right)$ is the initial value corresponding to $(\phi, \psi, w)$ (see (2.22)).

The function $(\phi, \psi, w)$ in Theorem 2.3 becomes a global solution of the problem (2.21), (2.22), which belongs to the space specified in Theorem 2.1. Thus we have a desired solution of the original problem (2.1), (2.9). On the other hand the solution of (2.1) is unique in the above space. Therefore the proof of Theorem 2.1 is completed.

From these considerations, it is sufficient to prove Theorem 2.3 for our purpose. The local existence of a small solution of $(2.27),(2.28)$ can be shown in the standard way (cf. [19]). So, for the proof of Theorem 2.3, it suffices to show the following a priori estimates, because the continuation argument used in the proof of Theorem 1.3 is also applicable to this case

Proposition 2.4. (a priori estimate). Let $(\Phi, \Psi, W) \in X(0, T)($ for some $T>0$ ) be a solution of the problem (2.27), (2.28). Put

$$
N(t)=\sup _{0 \leqq \tau \leqq t}\left\{\left\|\left(\Phi, \Psi, W /(\gamma-1)^{1 / 2}\right)(\tau)\right\|+\left\|\left(\phi, \psi, w /(\gamma-1)^{1 / 2}\right)(\tau)\right\|_{1}\right\}
$$

for $t \in[0, T]$. Then there exist positive constants $\delta_{3}, \varepsilon_{3}\left(\leqq \varepsilon_{0}\right)$ and $C_{3}$, which are independent of $T, \gamma \in(1,2]$ and $\left(v_{ \pm}, u_{ \pm}, \theta_{ \pm}\right)$, such that if $(\gamma-1)\left|v_{+}-v_{-}\right| \leqq \delta_{3}$ and $N(T)$ $\leqq \varepsilon_{3}$, then the following estimate holds for $t \in[0, T]$.

$$
\begin{aligned}
N(t)^{2} & +\int_{0}^{t}\left\|\left|V_{\xi}\right|^{1 / 2}\left(\Psi, W /(\gamma-1)^{1 / 2}\right)(\tau)\right\|^{2}+\|\phi(\tau)\|_{1}^{2} \\
& +\|(\psi, w)(\tau)\|_{2}^{2} d \tau \leqq C_{3}^{2} N_{0}^{2} .
\end{aligned}
$$

2.3 A Priori Estimate, $I$. Let $(\Phi, \Psi, W) \in X(0, T)$ (for some $T>0$ ) be a solution of the problem (2.27), (2.28) satisfying $N(T) \leqq \varepsilon_{0}$, where $\varepsilon_{0}$ is a constant defined in (2.15). In the following, we use the symbols $C, c, \delta, \ldots$ to denote the constants which may depend on $\underline{v}, \bar{v}, \bar{u}, \underline{\theta}$ and $\bar{\theta}$ but not on $T, \gamma \in(1,2]$ and $\left(v_{ \pm}, u_{ \pm}, \underline{\theta}\right)$. We first show the estimate for the $L^{2}$-norm of the solution.

Lemma 2.5. There are positive constants $\delta_{4}$ and $C$ such that if $(\gamma-1)\left|v_{+}-v_{-}\right| \leqq \delta_{4}$, then

$$
\begin{aligned}
& \left\|\left(\Phi, \Psi, W /(\gamma-1)^{1 / 2}\right)(t)\right\|^{2}+\int_{0}^{t}\left\|\left|V_{\xi}\right|^{1 / 2}\left(\Psi, W /(\gamma-1)^{1 / 2}\right)(\tau)\right\|^{2} \\
& \quad+\left\|\left(\psi, W_{\xi}\right)(\tau)\right\|^{2} d \tau-C(\gamma-1)\left|v_{+}-v_{-}\right| \int_{0}^{t}\|\phi(\tau)\|^{2} d \tau \\
& \leqq C\left\{\left\|\Phi_{0}, \Psi_{0}, W_{0} /(\gamma-1)^{1 / 2}\right\|^{2}+\int_{0}^{t} \int|\Psi|\left|F_{1}\right|+|W|\left|F_{2}\right| d \xi d \tau\right\}
\end{aligned}
$$


holds for $t \in[0, T]$.

Proof. Putting $k(V)=\left(b_{1}-s^{2} V\right)^{-1}$, we multiply the equations of $\Phi, \Psi$ and $W$ in (2.29) by $\Phi, k(V) V \Psi$ and $R k(V)^{2} W$, respectively, and calculate their sums. Then we have

$$
\begin{aligned}
E_{1}(\Phi, \Psi, W)_{t} & +E_{2}\left(\Psi, \Psi_{\xi}\right)+E_{3}\left(W, W_{\xi}\right)+G\left(\Psi, W, \Phi_{\xi}, W_{\xi}\right) \\
& +\{\cdots\}_{\xi}=k(V) V \Psi F_{1}+R k(V)^{2} W F_{2},
\end{aligned}
$$

where

$$
\begin{aligned}
E_{1}(\Phi, \Psi, W) & =\frac{1}{2}\left\{\Phi^{2}+k(V) V \Psi^{2}+\frac{R^{2}}{\gamma-1} k(V)^{2} W^{2}\right\}, \\
E_{2}\left(\Psi, \Psi_{\xi}\right) & =A \Psi^{2}+\mu k(V) \Psi_{\xi}^{2}+\mu k(V)_{\xi} \Psi \Psi_{\xi},
\end{aligned}
$$

with $A=\frac{1}{2}(k(V) V)_{\xi}+(\gamma-1) k(V) U_{\xi}$,

$$
\begin{aligned}
E_{3}\left(W, W_{\xi}\right)= & \frac{s R^{2}}{\gamma-1} k(V) k(V)_{\xi} W^{2}+\kappa R \frac{k(V)^{2}}{V} W_{\xi}^{2}, \\
G\left(\Psi, W, \Phi_{\xi}, W_{\xi}\right)= & \kappa(\gamma-1) \frac{k(V)^{2}}{V} U_{\xi} \Psi W_{\xi}+\kappa R\left(\frac{k(V)^{2}}{V}\right)_{\xi} W\left(W_{\xi}+\frac{\gamma-1}{R} U_{\xi} \Psi\right) \\
& +\kappa R \frac{k(V)}{V^{2}} \Theta_{\xi} W \Phi_{\xi},
\end{aligned}
$$

and $\{\cdots\}_{\xi}$ denotes the term which disappears after integration with respect to $\xi \in \mathbb{R}$. Here we have used the equality $k(V)_{\xi}+s k(V)^{2} U_{\xi}=0$ to simplify the term $\bar{\Psi} W$.

We shall estimate the functions $E_{1}, E_{2}, E_{3}$ and $G$ by using the properties of the traveling wave solution. We first note that

$$
p_{ \pm} \leqq k(V)^{-1}=b_{1}-s^{2} V \leqq p_{\mp} \text { for } s \lessgtr 0,
$$

where $p_{ \pm}=R \theta_{ \pm} / v_{ \pm}$. By virtue of this inequality, we have

$$
\begin{gathered}
c\left(\Phi^{2}+\Psi^{2}+\frac{W^{2}}{\gamma-1}\right) \leqq E_{1} \leqq C\left(\Phi^{2}+\Psi^{2}+\frac{W^{2}}{\gamma-1}\right), \\
E_{3} \geqq c\left(\left|V_{\xi}\right| \frac{W^{2}}{\gamma-1}+W_{\xi}^{2}\right),
\end{gathered}
$$

with some positive constants $c$ and $C$, where $k(V)_{\xi}=s^{2} k(V)^{2} V_{\xi}$ and $s V_{\xi}>0$ are used in (2.38). Next, for any $\alpha>0$, there is a constant $C_{\alpha}$ such that

$$
|G| \leqq \alpha\left(\left|V_{\xi}\right| \frac{W^{2}}{\gamma-1}+W_{\xi}^{2}\right)+C_{\alpha}(\gamma-1) \mid v_{+}-v_{-}\left\{\left|V_{\xi}\right|\left(\Psi^{2}+\frac{W^{2}}{\gamma-1}\right)+\Phi_{\xi}^{2}\right\},
$$

where we have used the inequalities in (2.19).

Finally we show that suitably small $(\gamma-1) \mid\left(v_{+}-v_{-} \mid\right.$, say $(\gamma-1)\left|v_{+}-v_{-}\right| \leqq \delta$, there is a constant $c=c(\delta)>0$ satisfying

$$
E_{2} \geqq c\left(\left|V_{\xi}\right| \Psi^{2}+\Psi_{\xi}^{2}\right)
$$


For this purpose it is sufficient to check the inequalities

$$
\inf _{\xi} A /\left|V_{\xi}\right|>0 \text { and } \sup _{\xi} D /\left|V_{\xi}\right|<0
$$

for suitably small $(\gamma-1)\left|v_{+}-v_{-}\right|$, where

$$
D=\mu\left\{\mu\left|k(V)_{\xi}\right|^{2}-4 A k(V)\right\}
$$

is the discriminant of $E_{2}$ with respect to $\left(\Psi, \Psi_{\xi}\right)$. By direct calculations, using (2.8), we have

$$
A /\left|V_{\xi}\right|=\frac{|s|}{2} k(V)^{2} \tilde{A}, \quad D /\left|V_{\xi}\right|=-\mu|s| k(V)^{3} \tilde{D},
$$

where

$$
\tilde{A}=b_{1}-2(\gamma-1) k(V)^{-1}, \quad \tilde{D}=b_{1}+s^{2} R \Theta k(V)+k(V)^{-1}-4(\gamma-1) k(V)^{-1},
$$

with $b_{1}=p_{ \pm}+s^{2} v_{ \pm}$. We estimate $\tilde{A}$ and $\tilde{D}$ by using the inequalities (2.36) and $\theta_{ \pm} \leqq \Theta \leqq \theta_{\mp}$ (for $s \gtrless 0$ ). By the relations in $(2.18)_{1,2}$ there is a constant $C$ such that for $s \gtrless 0$,

$$
s^{2} \geqq \gamma p_{\mp} / v_{ \pm}-C(\gamma-1)\left|v_{+}-v_{-}\right|, \quad \theta_{ \pm} \geqq \theta_{\mp}-C(\gamma-1)\left|v_{+}-v_{-}\right| .
$$

Taking into account these inequalities, we get the following estimates for $s \gtrless 0$.

$$
\begin{aligned}
& \tilde{A} \geqq\left(p_{\mp}+s^{2} v_{\mp}\right)-2(\gamma-1) p_{\mp} \geqq(3-\gamma) R \theta_{\mp} / v_{ \pm}-C(\gamma-1)\left|v_{+}-v_{-}\right|, \\
& \tilde{D} \geqq\left(p_{\mp}+s^{2} v_{\mp}\right)+s^{2} R \theta_{ \pm} / p_{\mp}-4(\gamma-1) p_{\mp} \\
& \quad \geqq(5-2 \gamma) R \theta_{\mp} / v_{ \pm}-C(\gamma-1)\left|v_{+}-v_{-}\right|,
\end{aligned}
$$

where $C$ is a constant. Choose $\delta$ so that $2 C \delta=R \underline{\theta} / \bar{v}$. Then we have $\widetilde{A}, \widetilde{D} \geqq R \underline{\theta} / 2 \bar{v}$ for $(\gamma-1)\left|v_{+}-v_{-}\right| \leqq \delta$. This inequality together with (2.36) implies (2.41), and consequently the desired estimate (2.40).

Now, let $\alpha$ in (2.39) be $\alpha=c / 4$ and put $\delta_{4}=\min \left\{\delta, c / 4 C_{\alpha}\right\}$. We then integrate (2.35) over $[0, t] \times \mathbb{R}$ and use (2.37) (2.40) to obtain the desired estimate (2.34) under the condition $(\gamma-1)\left|v_{+}-v_{-}\right| \leqq \delta_{4}$. Thus the proof of Lemma 2.5 is completed.

Next we estimate the $L^{2}\left(0, T ; L^{2}\right)$-norm of the derivative $\Phi_{\xi}=\phi$.

Lemma 2.6. There is a constant $C$ such that

$$
\begin{aligned}
& \|\phi(t)\|^{2}+\int_{0}^{t}\|\phi(\tau)\|^{2} d \tau-C\left\{\|\Psi(t)\|^{2}+\int_{0}^{t}\left\|\left|V_{\xi}\right|^{1 / 2} \Psi(\tau)\right\|^{2}+\left\|\left(\psi, W_{\xi}\right)(\tau)\right\|^{2} d \tau\right\} \\
& \leqq C\left\{\left\|\Psi_{0}\right\|^{2}+\left\|\phi_{0}\right\|^{2}+\int_{0}^{t} \int|\phi|\left|F_{1}\right| d \xi d \tau\right\}
\end{aligned}
$$

holds for $t \in[0, T]$.

Proof. Multiply the equations $\Phi$ and $\Psi$ in (2.29) by $V \Psi_{\xi}-V_{\xi} \Psi$ and $-V \Phi_{\xi}$, respectively. Next we apply $\partial_{\xi}$ to the equation of $\Phi$ in (2.29) and then multiply the 
resulting equation by $\mu \Phi_{\xi}$. Calculating their sums, we arrive at the equality

$$
\begin{aligned}
& \left(\frac{\mu}{2} \Phi_{\xi}^{2}-V \Phi_{\xi} \Psi\right)_{t}+\left(b_{1}-s^{2} V\right) \Phi_{\xi}^{2}-V_{\xi}\left(s \Phi_{\xi}+\Psi_{\xi}\right)-V \Psi_{\xi}^{2} \\
& -\Phi_{\xi}\left\{R W_{\xi}+(\gamma-1) U_{\xi} \Psi\right\}+\{\cdots\}_{\xi}=-V \Phi_{\xi} F_{1},
\end{aligned}
$$

from which follows the desired estimate (2.42) after integration with respect to $t$ and $\xi$. This completes the proof.

Finally we note the estimate for the $L^{2}\left(0, T ; L^{2}\right)$-norm of $w$ :

$$
\int_{0}^{t}\|w(\tau)\|^{2} d \tau-C\left\{\int_{0}^{t}\left\|\left|V_{\xi}\right|^{1 / 2} \Psi(\tau)\right\|^{2}+\left\|W_{\xi}(\tau)\right\|^{2} d \tau\right\} \leqq C \int_{0}^{t} \int\left|\psi^{2} w\right| d \xi d \tau,
$$

with some constant $C$. This is an easy consequence of (2.26).

2.4 A Priori Estimate, II. We proceed to estimate the $H^{1}$-norm of the quantities $(\phi, \psi, w)$. We first rewrite $(2.21)$ (see also (2.23)) in the form

$$
\left\{\begin{array}{l}
\phi_{t}-s \phi_{\xi}-\psi_{\xi}=0, \\
\psi_{t}-s \psi_{\xi}-\frac{1}{V}\left(b_{1}-s^{2} V\right) \phi_{\xi}+\frac{R}{V} w_{\xi}-\left(\frac{\mu}{V} \psi_{\xi}\right)_{\xi} \\
\quad-\left\{\frac{1}{V}\left(b_{1}-s^{2} V\right)\right\}_{\xi} \phi+\left(\frac{R}{V}\right)_{\xi} w=f_{1}, \\
\frac{R}{\gamma-1}\left(w_{t}-s w_{\xi}\right)+\left(b_{1}-s^{2} V\right) \psi_{\xi}-\left(\frac{\kappa}{V} w_{\xi}\right)_{\xi} \\
\quad+\left(\frac{\kappa}{V^{2}} \Theta_{\xi} \phi\right)_{\xi}-\frac{1}{V}\left\{\left(b_{1}-s^{2} V\right) \phi-R w+\mu \psi_{\xi}\right\} U_{\xi}=f_{2},
\end{array}\right.
$$

where $f_{1}$ and $f_{2}$ are nonlinear terms with respect to $(\phi, \psi, w)$ :

$$
\left\{\begin{aligned}
f_{1}= & -\left\{\frac{\phi}{V(V+\phi)}\left\{\left(b_{1}-s^{2} V\right) \phi-R w+\mu \psi_{\xi}\right\}\right\}_{\xi}, \\
f_{2}= & \frac{1}{V+\phi}\left\{\left(b_{1}-s^{2} V\right) \phi-R w+\mu \psi_{\xi}\right\}\left(\psi_{\xi}-\frac{1}{V} U_{\xi} \phi\right) \\
& -\left\{\frac{\kappa \phi}{V(V+\phi)}\left(w_{\xi}-\frac{1}{V} \Theta_{\xi} \phi\right)\right\}_{\xi} .
\end{aligned}\right.
$$

Compare (2.44) and (2.45) with (2.29) and (2.30), respectively.

The estimates for the $L^{2}$-norm of $\partial_{\xi}^{l}(\phi, \psi, w), l=0$ and 1 , are obtained in a similar way as in Lemma 2.5 .

Lemma 2.7. Let $l=0$ and 1 . There is a constant $C$ such that

$$
\begin{aligned}
& \left\|\partial_{\xi}^{l}\left(\phi, \psi, w /(\gamma-1)^{1 / 2}\right)(t)\right\|^{2}+\int_{0}^{t}\left\|\partial_{\xi}^{l+1}(\psi, w)(\tau)\right\|^{2} d \tau \\
& \quad-C \int_{0}^{t}\|(\phi, \psi, w)(\tau)\|_{l}^{2} d \tau \leqq C\left\{\left\|\partial_{\xi}^{l}\left(\phi_{0}, \psi_{0}, w_{0} /(\gamma-1)^{1 / 2}\right)\right\|^{2}+\int_{0}^{t} \int R_{l} d \xi d \tau\right\}
\end{aligned}
$$


holds for $t \in[0, T]$, where

$$
R_{l}= \begin{cases}|\psi|\left|f_{1}\right|+|w|\left|f_{2}\right| & \text { for } l=0, \\ \left|\psi_{\xi}, \psi_{\xi \xi}\right|\left|f_{1}\right|+\left|w_{\xi}, w_{\xi \xi}\right|\left|f_{2}\right| & \text { for } l=1 .\end{cases}
$$

Proof. Put $k(V)=\left(b_{1}-s^{2} V\right)^{-1}$. We apply $\partial_{\xi}^{l}(l=0,1)$ to $(2.44)$ to obtain the system for $\partial_{\xi}^{l}(\phi, \psi, w)$. Multiply the equations of $\partial_{\xi}^{l} \phi, \partial_{\xi}^{l} \psi$ and $\partial_{\xi}^{l} w$ by $\partial_{\xi}^{l} \phi, k(V) V \partial_{\xi}^{l} \psi$ and $R k(V)^{2} \partial_{\xi}^{l} w$, respectively. Calculating their sums and integrating the resulting equality over $[0, t] \times \mathbb{R}$, we can get the desired estimate $(2.46)_{l}$ in a similar way as in Lemma 2.5. We omit the details.

For the $L^{2}\left(0, T ; L^{2}\right)$-norm of $\phi_{\xi}$, we have the following

Lemma 2.8. There is a constant $C$ such that the following estimate holds for $t \in[0, T]$.

$$
\begin{aligned}
& \left\|\phi_{\xi}(t)\right\|^{2}+\int_{0}^{t}\left\|\phi_{\xi}(\tau)\right\|^{2} d \tau-C\left\{\|\psi(t)\|^{2}+\int_{0}^{t}\|(\psi, w)(\tau)\|_{1}^{2} d \tau\right\} \\
& \leqq C\left\{\left\|\psi_{0}\right\|^{2}+\left\|\phi_{0, \xi}\right\|^{2}+\int_{0}^{t} \int\left|\phi_{\xi} \| f_{1}\right| d \xi d \tau\right\} .
\end{aligned}
$$

Proof. Multiply the equations of $\phi$ and $\psi$ in (2.44) by $V \psi_{\xi}-V_{\xi} \psi$ and $-V \phi_{\xi}$, respectively. Next we apply $\partial_{\xi}$ to the equation of $\phi$ in (2.44) and then multiply the resulting equation by $\mu \phi_{\xi}$. Calculating their sums and integrating over $[0, t] \times \mathbb{R}$, we can get the desired estimate (2.47) similarly as in Lemma 2.6. We omit the details.

Now, we shall combine the estimates $(2.34),(2.42),(2.43),(2.46)_{1},(2.46)_{2}$ and (2.47) to get the desired estimate (2.33). Firstly, we calculate $(2.34)+\{(2.42)+(2.43)\} \times \alpha$ with $\alpha=1 / 4 C$. Then, for $(\gamma-1)\left|v_{+}-v_{-}\right| \leqq \delta_{3} \equiv \min \left\{\delta_{4}, \alpha / 2 C\right\}$, we have

$$
\begin{aligned}
\|(\Phi, & \left.\Psi, W /(\gamma-1)^{1 / 2}\right)(t)\left\|^{2}+\right\| \phi(t) \|^{2} \\
& +\int_{0}^{t}\left\|\left|V_{\xi}\right|^{1 / 2}\left(\Psi, W /(\gamma-1)^{1 / 2}\right)(\tau)\right\|^{2}+\|(\phi, \psi, w)(\tau)\|^{2} d \tau \\
\leqq & C\left\{\left\|\Phi_{0}, \Psi_{0}, W_{0} /(\gamma-1)^{1 / 2}\right\|^{2}+\left\|\phi_{0}\right\|^{2}\right. \\
& \left.+\int_{0}^{t} \int|\Psi, \phi|\left|F_{1}\right|+|W|\left|F_{2}\right|+\left|\psi^{2} w\right| d \xi d \tau\right\} .
\end{aligned}
$$

Combining successively the estimates $(2.48),(2.46)_{1},(2.47)$ and $(2.46)_{2}$, we reach the following inequality.

$$
\begin{aligned}
& N(t)^{2}+\int_{0}^{t}\left\|\left|V_{\xi}\right|^{1 / 2}\left(\Psi, W /(\gamma-1)^{1 / 2}\right)(\tau)\right\|^{2}+\|\phi(\tau)\|_{1}^{2}+\|(\psi, w)(\tau)\|_{2}^{2} d \tau \\
& \leqq C \\
& \quad\left\{N_{0}^{2}+\int_{0}^{t} \int|\Psi, \phi|\left|F_{1}\right|+|W|\left|F_{2}\right|+\left|\psi^{2} w\right|+\left|\psi, \phi_{\xi}, \psi_{\xi}, \psi_{\xi \xi}\right|\left|f_{1}\right|\right. \\
& \left.\quad+\left|w, w_{\xi}, w_{\xi \xi}\right|\left|f_{2}\right| d \xi d \tau\right\} .
\end{aligned}
$$

It follows from (2.30), (2.45) and (2.19) that for $|\phi, \psi, w| \rightarrow 0$,

$$
\begin{aligned}
\left|F_{1}, F_{2}\right| & =O\left(|\phi, \psi, w|^{2}+|\phi, \psi|\left|\psi_{\xi}, w_{\xi}\right|\right), \\
\left|f_{1}, f_{2}\right| & =O\left(|\phi, w|^{2}+|\phi, w|\left|\phi_{\xi}, \psi_{\xi}, w_{\xi}\right|+\left|\phi_{\xi}, \psi_{\xi}\right|\left|\psi_{\xi}, w_{\xi}\right|+|\phi|\left|\psi_{\xi \xi}, w_{\xi \xi}\right|\right) .
\end{aligned}
$$


Therefore the integral on the right-hand side of (2.49) is dominated by

$$
C N(t) \int_{0}^{t}\|\phi(\tau)\|_{1}^{2}+\|(\psi, w)(\tau)\|_{2}^{2} d \tau
$$

with some constant $C=C\left(\varepsilon_{0}\right)$, where $N(T) \leqq \varepsilon_{0}$ is assumed. Thus we arrive at the desired estimate (2.33) for suitably small $N(T)$. Thus the proof of Proposition 2.4 is completed.

\section{The Broadwell Model System}

3.1 Traveling Wave Solution and Main Theorem. The one-dimensional Broadwell model system for a simple discrete velocity gas is written in the form (cf. [1])

$$
\left\{\begin{array}{l}
F_{1, t}+v F_{1, x}=F_{2}^{2}-F_{1} F_{3}, \\
F_{2, t}=-\frac{1}{2}\left(F_{2}^{2}-F_{1} F_{3}\right), \\
F_{3, t}-v F_{3, x}=F_{2}^{2}-F_{1} F_{3},
\end{array}\right.
$$

where the unknown functions $F_{1}, F_{2}$ and $F_{3}$ represent the mass densities for gas particles moving in $x$-direction with the constant speeds $v, 0$ and $-v$, respectively. Here $v$ is a positive constant.

For the density distribution function $F=\left(F_{1}, F_{2}, F_{3}\right)$, we define the hydrodynamical moments, the mass density $\rho$ and the momentum $m$ (in $x$-direction), by

$$
\rho=F_{1}+4 F_{2}+F_{3}, \quad m=v\left(F_{1}-F_{3}\right) .
$$

We also define another quantity $z$ by

$$
z=v^{2}\left(F_{1}+F_{3}\right) \text {. }
$$

Then $F=\left(F_{1}, F_{2}, F_{3}\right)$ is conversely represented by $(\rho, m, z)$ as follows:

$$
\begin{aligned}
& F_{1}=\left(z+v^{2} m\right) / 2 v^{2}, \\
& F_{2}=\left(v^{2} \rho-z\right) / 4 v^{2}, \\
& F_{3}=\left(z-v^{2} m\right) / 2 v^{2} .
\end{aligned}
$$

Using (3.3), we can transform (3.1) into the following equivalent system.

$$
\begin{aligned}
& \rho_{t}+m_{x}=0, \\
& m_{t}+z_{x}=0, \\
& z_{t}+v^{2} m_{x}=\left\{\left(v^{2} \rho-z\right)^{2}-4\left(z^{2}-v^{2} m^{2}\right)\right\} / 8 v^{2} .
\end{aligned}
$$

The function $F=\left(F_{1}, F_{2}, F_{3}\right)$ satisfying the conditions $F_{1}, F_{2}, F_{3}>0$ (positiveness) and $F_{2}^{2}=F_{1} F_{3}$ is called a local Maxwellian. By the relations in (3.3), the condition for the positiveness of $F$ is equivalent to

$$
v|m|<z<v^{2} \rho
$$

and the condition for $F$ to be a local Maxwellian is

$$
\rho>0, \quad|u|<v, \quad z=\rho \sigma(u),
$$


where $u=m / \rho$ is the fluid velocity, and

$$
\sigma(u)=\frac{v^{2}}{3}\left\{2\left(1+3 u^{2} / v^{2}\right)^{1 / 2}-1\right\} .
$$

In this paper, the function $(\rho, m, z)$ satisfying condition (3.6) is also called a local Maxwellian.

In what follows, we shall investigate the system (3.4) instead of the original system (3.1). The system (3.4) admits smooth traveling wave solutions with shock profile

$$
\begin{aligned}
& (\rho, m, z)(t, x)=(P, M, Z)(\xi), \quad \xi=x-s t, \\
& (P, M, Z)(\xi) \rightarrow\left(\rho_{ \pm}, m_{ \pm}, z_{ \pm}\right) \quad \text { as } \quad \xi \rightarrow \pm \infty,
\end{aligned}
$$

where $\rho_{ \pm}, m_{ \pm}, z_{ \pm}$and $s$ are given constants satisfying the condition (3.6), i.e.,

$$
\rho_{ \pm}>0, \quad\left|u_{ \pm}\right|<v, \quad z_{ \pm}=\rho_{ \pm} \sigma\left(u_{ \pm}\right) \quad\left(\text { with } u_{ \pm}=m_{ \pm} / \rho_{ \pm}\right)
$$

the Rankine--Hugoniot condition

$$
\left\{\begin{array}{l}
-s\left(\rho_{+}-\rho_{-}\right)+\left(m_{+}-m_{-}\right)=0 \\
-s\left(m_{+}-m_{-}\right)+\left(z_{+}-z_{-}\right)=0
\end{array}\right.
$$

and in addition Lax's shock condition that

$$
\text { either } \lambda_{-}\left(u_{+}\right)<s<\lambda_{-}\left(u_{-}\right) \text {or } \lambda_{+}\left(u_{+}\right)<s<\lambda_{+}\left(u_{-}\right) \text {. }
$$

Here $\lambda_{ \pm}(u)=\left(u \pm \sigma(u)^{1 / 2}\right) / \sigma_{0}(u)$ with $\sigma_{0}(u)=\left(1+3 u^{2} / v^{2}\right)^{1 / 2}$ are the eigenvalues of the hyperbolic system

$$
\left\{\begin{array}{l}
\rho_{t}+m_{x}=0, \\
m_{t}+\{\rho \sigma(u)\}_{x}=0, \quad u=m / \rho,
\end{array}\right.
$$

which is derived from (3.1) (or (3.4)) by the Chapman-Enskog expansion. Note that condition (3.12) is equivalent to (see [1])

$$
u_{+}<u_{-} \quad\left(\text { or } s\left(\rho_{+}-\rho_{-}\right)<0\right) \text {. }
$$

Let $\rho<\bar{\rho}$ and $\bar{u}<v$ be any fixed positive constants. We simply assume that the constants $\rho_{ \pm}, m_{ \pm}$and $z_{ \pm}$in (3.10) (3.12) lie in the region

$$
\underline{\rho} \leqq \rho_{ \pm} \leqq \bar{\rho}, \quad\left|u_{ \pm}\right| \leqq \bar{u} \quad\left(\text { with } u_{ \pm}=m_{ \pm} / \rho_{ \pm}\right) .
$$

We consider the initial value problem for (3.4) in a neighborhood of a traveling wave solution. Let $(P, M, Z)(\xi), \xi=x-s t$, be a traveling wave solution. Suppose that the initial data

$$
(\rho, m, z)(0, x)=\left(\rho_{0}, m_{0}, z_{0}\right)(x)
$$

satisfy

$$
\left(\rho_{0}-P, m_{0}-M, z_{0}-Z\right) \in H^{1}
$$


Moreover we assume that the integrals

$$
\Phi_{0}(x)=\int_{-\infty}^{x}\left(\rho_{0}-P\right)(y) d y, \quad \Psi_{0}(x)=\int_{-\infty}^{x}\left(m_{0}-M\right)(y) d y
$$

exist for any $x \in \mathbb{R}$, and satisfy

$$
\left(\Phi_{0}, \Psi_{0}\right) \in L^{2} .
$$

Note that the assumptions (3.15) and (3.17) imply the relations

$$
\int_{-\infty}^{+\infty}\left(\rho_{0}-P\right)(x) d x=0, \quad \int_{-\infty}^{+\infty}\left(m_{0}-M\right)(x) d x=0
$$

Our main theorem in this section is

Theorem 3.1. Let $\rho_{ \pm}, m_{ \pm}, z_{ \pm}$and $s$ be given constants satisfying $(3.10) \sim(3.13)$, and let $(P, M, Z)(\xi), \xi=x-$ st, be a traveling wave solution which smoothly interpolates the asymptotic values $\left(\rho_{ \pm}, m_{ \pm}, z_{+}\right)$with the speed $s$. Suppose that the initial data $\left(\rho_{0}, m_{0}, z_{0}\right)$ satisfy (3.15) and (3.17). Then there exist positive constants $\delta_{0}$ and $\varepsilon_{0}$ independent of $\left(\rho_{ \pm}, m_{ \pm}, z_{ \pm}\right)$such that if $\left|\rho_{+}-\rho_{-}\right| \leqq \delta_{0}$ and

$$
N_{0}=\left\|\rho_{0}-P, m_{0}-M, z_{0}-Z\right\|_{1}+\left\|\Phi_{0}, \Psi_{0}\right\| \leqq \varepsilon_{0},
$$

then the initial value problem (3.4), (3.14) has a unique global solution $(\rho, m, z)(t, x)$, which satisfies

$$
(\rho-P, m-M, z-Z) \in C^{0}\left(0, \infty ; H^{1}\right) \cap L^{2}\left(0, \infty ; H^{1}\right),
$$

and asymptotically tends to the traveling wave solution in the maximum norm:

$$
\sup _{x \in \mathbb{R}}|(\rho, m, z)(t, x)-(P, M, Z)(x-s t)| \rightarrow 0 \quad \text { as } \quad t \rightarrow \infty .
$$

It is worth noting that the traveling wave solution $(P, M, Z)(\xi)$ satisfies the condition (3.5) uniformly in $\xi \in \mathbb{R}$ (see (3.26)), and so does the solution $(\rho, m, z)(t, x)$, provided $N_{0}$ is suitably small. Therefore we get a positive solution of the original system (3.1) through the relation (3.3).

For the proof of Theorem 3.1, we first study the properties of traveling wave solutions. The eigenvalues $\lambda_{ \pm}(u)$ satisfy (cf. [1])

$$
\frac{d}{d u} \lambda_{ \pm}(u)>0, \quad \lambda_{-}(u)<\min \{u, 0\} \leqq \max \{u, 0\}<\lambda_{+}(u)
$$

for $|u|<v$. Therefore, noting the equalities $\lambda_{-}(-v)=-v, \lambda_{-}(v)=\lambda_{+}(-v)=0$ and $\lambda_{+}(v)=v$, we have

$$
-v+c \leqq \lambda_{-}(u) \leqq \min \{u, 0\}-c, \quad \max \{u, 0\}+c \leqq \lambda_{+}(u) \leqq v-c
$$

for $|u| \leqq \bar{u}$, where $c=c(\bar{u})(<v)$ is a positive constant. In particular, from (3.12) and (3.13), we have

$$
-v+c \leqq s \leqq \min \left\{u_{-}, 0\right\}-c \text { or } \max \left\{u_{+}, 0\right\}+c \leqq s \leqq v-c .
$$


The system for the traveling wave solution $(P, M, Z)(\xi)$ is

$$
\left\{\begin{array}{l}
-s P_{\xi}+M_{\xi}=0, \\
-s M_{\xi}+Z_{\xi}=0, \\
-s Z_{\xi}+v^{2} M_{\xi}=\left\{\left(v^{2} P-Z\right)^{2}-4\left(Z^{2}-v^{2} M^{2}\right)\right\} / 8 v^{2} .
\end{array}\right.
$$

The first two equations give

$$
-s P+M=a_{1}, \quad-s M+Z=a_{2},
$$

where $a_{1}=-s \rho_{ \pm}+m_{ \pm}$and $a_{2}=-s m_{ \pm}+z_{ \pm}$are the integral constants. Substituting (3.23) $)_{1}$ into the third equation of (3.22), we obtain

$$
s P_{\xi}=\frac{v^{2}+3 s^{2}}{8 v^{2}}\left(P-\rho_{+}\right)\left(P-\rho_{-}\right),
$$

where $|s|<v$ is used. The existence and uniqueness (up to a shift in $\xi$ ) of the solution of (3.22) with the condition (3.9) is obvious from (3.23) $)_{1,2}$ and (3.12)'.

We summarize the properties of the traveling wave solution.

Lemma 3.2. The traveling wave solution $(P, M, Z)(\xi)$ satisfies

$$
s P_{\xi}<0, \quad M_{\xi}=s P_{\xi}<0, \quad s Z_{\xi}=s^{3} P_{\xi}<0
$$

for any $\xi \in \mathbb{R}$. Moreover there are positive constants $C$ and $c$ depending only on $\underline{\rho}, \bar{\rho}$ and $\bar{u}$ such that for $\xi \in \mathbb{R}$,

$$
\begin{gathered}
\left|P_{\xi}\right| \leqq C\left|\rho_{+}-\rho_{-}\right|^{2}, \\
v|M|+c \leqq Z \leqq v^{2} P-c .
\end{gathered}
$$

The inequalities in (3.24) and (3.25) are obvious. The estimate $v M+c \leqq Z$ follows from the fact that $Z-v M$ is a monotone function of $\xi \in \mathbb{R}$ with the asymptotic values $z_{ \pm}-v m_{ \pm} \geqq z_{ \pm}-v\left|m_{+}\right| \geqq c$ for $\xi \rightarrow \pm \infty$, where $c$ is a positive constant depending only on $\rho, \bar{\rho}$ and $\bar{u}$. Similarly, we have $-v M+c \leqq Z$ and $Z \leqq v^{2} P-c$ with some positive constant $c$. This completes the proof of (3.26).

3.2. Reformulation of the Problem. Let us reset the problem (3.4), (3.14) on the moving coordinate $\xi=x-$ st. Putting

$$
(\rho, m, z)(t, x)=(P, M, Z)(\xi)+(\phi, \psi, w)(t, \xi),
$$

we rewrite the problem (3.4), (3.14) in the form

$$
\begin{gathered}
\left\{\begin{array}{l}
\phi_{t}-s \phi_{\xi}+\psi_{\xi}=0, \\
\psi_{t}-s \psi_{\xi}+w_{\xi}=0, \\
w_{t}-s w_{\xi}+v^{2} \psi_{\xi}-A \phi-M \psi+B w=\Gamma(\phi, \psi, w),
\end{array}\right. \\
(\phi, \psi, w)(0, \xi)=\left(\phi_{0}, \psi_{0}, w_{0}\right)(\xi) \equiv\left(\rho_{0}-P, m_{0}-M, z_{0}-Z\right)(\xi),
\end{gathered}
$$

where

$$
\begin{gathered}
A=\left(v^{2} P-Z\right) / 4, \quad B=\left(v^{2} P+3 Z\right) / 4 v^{2}, \\
\Gamma(\phi, \psi, w)=\left\{\left(v^{2} \phi-w\right)^{2}-4\left(w^{2}-v^{2} \psi^{2}\right)\right\} / 8 v^{2} .
\end{gathered}
$$


Inspired by the relations $\left(\phi_{0}, \psi_{0}\right)=\left(\Phi_{0, \xi}, \Psi_{0, \xi}\right)$ (see (3.16)), we seek the solution of the problem (3.28), (3.29) in the form

$$
(\phi, \psi, w)=\left(\Phi_{\xi}, \Psi_{\xi}, w\right)
$$

Substituting (3.32) into (3.28) and integrating the first two equations once with respect to $\xi$, we get

$$
\left\{\begin{array}{l}
\Phi_{t}-s \Phi_{\xi}+\Psi_{\xi}=0 \\
\Psi_{t}-s \Psi_{\xi}+w=0 \\
w_{t}-s w_{\xi}+v^{2} \Psi_{\xi \xi}-A \Phi_{\xi}-M \Psi_{\xi}+B w=\Gamma\left(\Phi_{\xi} \Psi_{\xi}, w\right) .
\end{array}\right.
$$

The second equation of (3.33) gives

$$
w=-\left(\Psi_{t}-s \Psi_{\xi}\right)
$$

Substituting (3.34) into the remaining equations of (3.33), we get a closed system for $(\Phi, \Psi)$ :

$$
\begin{aligned}
L_{1}(\Phi, \Psi) \equiv & \Phi_{t}-s \Phi_{\xi}+\Psi_{\xi}=0, \\
L_{2}(\Phi, \Psi) \equiv & \left(\Psi_{t}-s \Psi_{\xi}\right)_{t}-s\left(\Psi_{t}-s \Psi_{\xi}\right)_{\xi}-v^{2} \Psi_{\xi \xi} \\
& +A \Phi_{\xi}+(M-s B) \Psi_{\xi}+B \Psi_{t}=-\Gamma\left(\Phi_{\xi}, \Psi_{\xi},-\left(\Psi_{t}-s \Psi_{\xi}\right)\right),
\end{aligned}
$$

with the initial condition

$$
(\Phi, \Psi)(0, \xi)=\left(\Phi_{0}, \Psi_{0}\right)(\xi), \quad \Psi_{t}(0, \xi)=\Psi_{1}(\xi) \equiv\left(s \Psi_{0, \xi}-w_{0}\right)(\xi)
$$

We introduce the solution space of the problem (3.35), (3.36) as follows.

$$
\begin{aligned}
X(0, T)= & \left\{(\Phi, \Psi)(t, \xi) ; \quad \Phi \in C^{0}\left(0, T ; H^{2}\right), \quad \Psi \in C^{0}\left(0, T ; H^{2}\right) \cap C^{1}\left(0, T ; H^{1}\right),\right. \\
& \left.\cdot\left(\Phi_{\xi}, \Psi_{\xi}, \Psi_{t}\right) \in L^{2}\left(0, T ; H^{1}\right)\right\} .
\end{aligned}
$$

Then we have the following

Theorem 3.3. Suppose that $\left(\Phi_{0}, \Psi_{0}\right) \in H^{2}$ and $\Psi_{1} \in H^{1}$. Then there exist positive constants $\delta_{1}, \varepsilon_{1}$ and $C_{1}$ independent of $\left(\rho_{ \pm}, m_{ \pm}, z_{ \pm}\right)$such that if $\left|\rho_{+}-\rho_{-}\right| \leqq \delta_{1}$ and

$$
N_{0}=\left\|\Phi_{0}, \Psi_{0}\right\|_{2}+\left\|w_{0}\right\|_{1} \leqq \varepsilon_{1} \quad\left(w_{0}=-\left(\Psi_{1}-s \Psi_{0, \xi}\right)\right),
$$

then the problem (3.35), (3.36) has a unique global solution $(\Phi, \Psi) \in X(0,+\infty)$ satisfying

$$
\|(\Phi, \Psi)(t)\|_{2}^{2}+\left\|\Psi_{t}(t)\right\|_{1}^{2}+\int_{0}^{t}\left\|\left(\Phi_{\xi}, \Psi_{\xi}, \Psi_{t}\right)(\tau)\right\|_{1}^{2} d \tau \leqq C_{1}^{2} N_{0}^{2}
$$

for $t \in[0, \infty)$.

For the solution $(\Phi, \Psi)$ in the above theorem, we define $(\phi, \psi, w)$ by $(3.32)$ and (3.34). Then it becomes a global solution of the problem (3.28), (3.29), and consequently we have the desired solution of the problem (3.4), (3.14) through the relation (3.27). On the other hand the solution of (3.4) is unique in the space $C^{0}\left(0, T ; H^{1}\right)$. Therefore the proof of Theorem 3.1 is completed.

To check the local existence of a solution of (3.35), we transform (3.35) into the 
following system of third order.

$$
\left(\partial_{t}-s \partial_{\xi}\right)\left(\partial_{t}-(s+v) \partial_{\xi}\right)\left(\partial_{t}-(s-v) \partial_{\xi}\right)\left(\begin{array}{c}
\Phi \\
\Psi
\end{array}\right)+\text { lower order terms }=0 .
$$

This system is strictly hyperbolic, and therefore the standard arguments give a solution $(\Phi, \Psi) \in X(0, T)$ for some $T>0$. So, for the proof of Theorem 3.3, it is sufficient to show the following a priori estimates.

Proposition 3.4 (a priori estimate). Suppose that the problem (3.35), (3.36) has a solution $(\Phi, \Psi) \in X(0, T)$ for some $T>0$. Put

$$
N(t)=\sup _{0 \leqq}\left\{\|(\Phi, \Psi)(\tau)\|_{2}+\left\|\Psi_{t}(\tau)\right\|_{1}\right\}
$$

for $t \in[0, T]$. Then there exist positive constants $\delta_{2}, \varepsilon_{2}$ and $C_{2}$ independent of $T$ and $\left(\rho_{ \pm}, m_{ \pm}, z_{ \pm}\right)$such that if $\left|\rho_{+}-\rho_{-}\right| \leqq \delta_{2}$ and $N(T) \leqq \varepsilon_{2}$, then

$$
N(t)^{2}+\int_{0}^{t}\left\|\left(\Phi_{\xi}, \Psi_{\xi}, \Psi_{t}\right)(\tau)\right\|_{1}^{2} d \tau \leqq C_{2}^{2} N_{0}^{2}
$$

holds for $t \in[0, T]$.

3.3. A Priori Estimate. Throughout this subsection we suppose that the problem (3.35), (3.36) has a solution $(\Phi, \Psi) \in X(0, T)$ for some $T>0$. As in Sect. 2, the symbols $C, c$ and $\delta$ denote the constants with may depend on $\rho, \bar{\rho}$ and $\bar{u}$ but not on $T$ and $\left(\rho_{ \pm}, m_{+}, z_{+}\right)$.

First we show the following basic estimates.

Lemma 3.5. There are positive constants $\delta$ and $C$ such that if $\left|\rho_{+}-\rho_{-}\right| \leqq \delta$, then

$$
\begin{gathered}
\|\Phi(t)\|^{2}+\|\Psi(t)\|_{1}^{2}+\left\|\Psi_{t}(t)\right\|^{2}+\int_{0}^{t}\left\|\left|P_{\xi}\right|^{1 / 2} \Psi(\tau)\right\|^{2}+\left\|\left(\Psi_{\xi}, \Psi_{t}\right)(\tau)\right\|^{2} d \tau \\
\leqq C\left\{\left\|\Phi_{0}\right\|^{2}+\left\|\Psi_{0}\right\|_{1}^{2}+\left\|w_{0}\right\|^{2}+\int_{0}^{t} \int\left(|\Psi|+\left|\Psi_{\xi}, \Psi_{t}\right|\right)|\Gamma| d \xi d \tau\right\}
\end{gathered}
$$

holds for $t \in[0, T]$.

Proof. We first note the elemental estimates for the coefficients $A, M$ and $B$ in Eqs. (3.35). From (3.24) we know that

$$
s A_{\xi}=\frac{1}{4}\left(v^{2}-s^{2}\right) s P_{\xi}<0, \quad s B_{\xi}=\frac{1}{4 v^{2}}\left(v^{2}+3 s^{2}\right) s P_{\xi}<0,
$$

where $|s|<v$ is used. Therefore there are positive constants $c$ and $C$ such that for $s \gtrless 0$,

$$
c \leqq A_{ \pm} \leqq A \leqq A_{\mp} \leqq C, \quad c \leqq B_{ \pm} \leqq B \leqq B_{\mp} \leqq C,
$$

where $A_{ \pm}\left(v^{2} \rho_{ \pm}-z_{ \pm}\right) / 4$ and $B_{ \pm}=\left(v^{2} \rho_{ \pm}+3 z_{ \pm}\right) / 4 v^{2}$. We also know that

$$
\left\{A^{-1}(M-s B)\right\}_{\xi}=\frac{1}{4}\left(v^{2}-s^{2}\right) A^{-2} a_{1} P_{\xi}<0,
$$

where $|s|<v$ and $s a_{1}=-s \rho_{ \pm}\left(s-u_{ \pm}\right)<0($ see $(3.21))$ are used. Therefore we have the 
estimate

$$
-\left\{A^{-1}(M-s B)\right\}_{\xi} \geqq c\left|P_{\xi}\right|
$$

with some positive constant $c$.

Now we show (3.40). Firstly, we calculate

$$
-\Psi_{\xi} \cdot L_{1}+A^{-1}\left(\Psi_{t}-s \Psi_{\xi}\right) \cdot L_{2}=-A^{-1}\left(\Psi_{t}-s \Psi_{\xi}\right) \cdot \Gamma
$$

The left-hand side is reduced to

$$
\begin{aligned}
& A^{-1}\left\{\frac{1}{2}\left(\Psi_{t}-s \Psi_{\xi}\right)^{2}+\frac{v^{2}}{2} \Psi_{\xi}^{2}-A \Phi \Psi_{\xi}\right\}_{t} \\
& \quad+A^{-1}\left\{B\left(\Psi_{t}-s \Psi_{\xi}\right)^{2}+M\left(\Psi_{t}-s \Psi_{\xi}\right) \Psi_{\xi}-A \Psi_{\xi}^{2}\right\} \\
& \quad+\frac{1}{2}\left(A^{-1}\right)_{\xi}\left\{s\left(\Psi_{t}-s \Psi_{\xi}\right)^{2}+2 v^{2}\left(\Psi_{t}-s \Psi_{\xi}\right) \Psi_{\xi}+s v^{2} \Psi_{\xi}^{2}\right\}+\{\cdots\}_{\xi},
\end{aligned}
$$

where $\{\cdots\}_{\xi}$ denotes the term which disappears after integration with respect to $\xi \in \mathbb{R}$. Secondly, we calculate

$$
\Phi \cdot L_{1}+A^{-1} \Psi \cdot L_{2}=-A^{-1} \Psi \cdot \Gamma
$$

The left member is reduced to

$$
\begin{aligned}
& {\left[A^{-1}\left\{\frac{1}{2} A \Phi^{2}+\frac{1}{2} B \Psi^{2}+\Psi\left(\Psi_{t}-s \Psi_{\xi}\right)\right\}+\frac{s}{2}\left(A^{-1}\right)_{\xi} \Psi^{2}\right]_{t}} \\
& \quad+A^{-1}\left\{-\left(\Psi_{t}-s \Psi_{\xi}\right)^{2}+v^{2} \Psi_{\xi}^{2}\right\}-\frac{1}{2}\left\{A^{-1}(M-s B)\right\}_{\xi} \Psi^{2} \\
& \quad+\left(v^{2}-s^{2}\right)\left(A^{-1}\right)_{\xi} \Psi \Psi_{\xi}+\{\cdots\}_{\xi} .
\end{aligned}
$$

Hence, the combination $(3.43)+(3.44) \times \lambda$ with a positive constant $\lambda$ yields

$$
\begin{aligned}
&\left\{E_{1}\left(\Phi, \Psi_{\xi}\right)\right.\left.+E_{2}\left(\Psi, \Psi_{t}-s \Psi_{\xi}\right)+\widetilde{E}_{2}(\Psi)\right\}_{t} \\
&+E_{3}\left(\Psi_{t}-s \Psi_{\xi}, \Psi_{\xi}\right)+E_{4}(\Psi)+G\left(\Psi, \Psi_{t}-s \Psi_{\xi}, \Psi_{\xi}\right)+\{\cdots\}_{\xi} \\
&=-A^{-1}\left\{\lambda \Psi+\left(\Psi_{t}-s \Psi_{\xi}\right)\right\} \cdot \Gamma
\end{aligned}
$$

where

$$
\begin{aligned}
E_{1}\left(\Phi, \Psi_{\xi}\right) & =A^{-1}\left(\frac{\lambda}{2} A \Phi^{2}-A \Phi \Psi_{\xi}+\frac{v^{2}}{2} \Psi_{\xi}^{2}\right), \\
E_{2}\left(\Psi, \Psi_{t}-s \Psi_{\xi}\right) & =A^{-1}\left\{\frac{\lambda}{2} B \Psi^{2}+\lambda \Psi\left(\Psi_{t}-s \Psi_{\xi}\right)+\frac{1}{2}\left(\Psi_{t}-s \Psi_{\xi}\right)^{2}\right\}, \\
E_{3}\left(\Psi_{t}-s \Psi_{\xi}, \Psi_{\xi}\right) & =A^{-1}\left\{(B-\lambda)\left(\Psi_{t}-s \Psi_{\xi}\right)^{2}+M\left(\Psi_{t}-s \Psi_{\xi}\right) \Psi_{\xi}+\left(\lambda v^{2}-A\right) \Psi_{\xi}^{2}\right\}, \\
\tilde{E}_{2}(\Psi) & =\frac{\lambda s}{2}\left(A^{-1}\right)_{\xi} \Psi^{2}, \quad E_{4}(\Psi)=-\frac{\lambda}{2}\left\{A^{-1}(M-s B)\right\}_{\xi} \Psi^{2},
\end{aligned}
$$

$G\left(\Psi, \Psi_{t}-s \Psi_{\xi}, \Psi_{\xi}\right)=\lambda\left(v^{2}-s^{2}\right)\left(A^{-1}\right)_{\xi} \Psi \Psi_{\xi}$

$$
+\frac{1}{2}\left(A^{-1}\right)_{\xi}\left\{s\left(\Psi_{t}-s \Psi_{\xi}\right)^{2}+2 v^{2}\left(\Psi_{t}-s \Psi_{\xi}\right) \Psi_{\xi}+s v^{2} \Psi_{\xi}^{2}\right\} .
$$

We choose a constant $\lambda>0$ so that the inequalities

$$
\sup _{\xi} D_{j}<0, \quad j=1,2,3 \text {, }
$$


hold uniformly in $\left(\rho_{ \pm}, m_{ \pm}, z_{ \pm}\right)$, where $D_{j}$ is the discriminant of the function $E_{j}$ :

$$
D_{1}=A\left(A-\lambda v^{2}\right), \quad D_{2}=\lambda(\lambda-B), \quad D_{3}=M^{2}-4\left(A-\lambda v^{2}\right)(\lambda-B) .
$$

For this choice of $\lambda$, there exist positive constants $c$ and $C$ such that

$$
\left\{\begin{array}{l}
c\left(\Phi^{2}+\Psi_{\xi}^{2}\right) \leqq E_{1} \leqq C\left(\Phi^{2}+\Psi_{\xi}^{2}\right) \\
c\left\{\Psi^{2}+\left(\Psi_{t}-s \Psi_{\xi}\right)^{2}\right\} \leqq E_{2} \leqq C\left\{\Psi^{2}+\left(\Psi_{t}-s \Psi_{\xi}\right)^{2}\right\} \\
E_{3} \geqq c\left\{\left(\Psi_{t}-s \Psi_{\xi}\right)^{2}+\Psi_{\xi}^{2}\right\}
\end{array}\right.
$$

On the other hand, (3.41) and (3.42) give

$$
0 \leqq \widetilde{E}_{2} \leqq C \Psi^{2} \quad \text { and } \quad E_{4} \geqq c\left|P_{\xi}\right| \Psi^{2},
$$

respectively, where $C$ and $c$ are positive constants. Moreover, for any $\alpha>0$, we have

$$
|G| \leqq \alpha\left|P_{\xi}\right| \Psi^{2}+C_{\alpha}\left|\rho_{+}-\rho_{-}\right|^{2}\left\{\left(\Psi_{t}-s \Psi_{\xi}\right)^{2}+\Psi_{\xi}^{2}\right\}
$$

with some constant $C_{\alpha}$, where (3.25) is used. We choose $\alpha$ so that $\alpha=c / 2$. For this choice of $\alpha$, we assume that $\left|\rho_{+}-\rho_{-}\right|^{2} \leqq c / 2 C_{\alpha}$. Then the equality (3.45) together with the estimates $(3.47) \sim(3.49)$ give the desired estimate (3.40) after integration with respect to $t$ and $\xi$,

It remains to check condition (3.46). It is easily seen from $\left(3.41^{\prime}\right)$ that the first two inequalities in (3.46) are equivalent to

$$
A_{\mp} / v^{2}<\lambda<B_{ \pm} \quad(\text { for } s \gtrless 0) \text {. }
$$

On the other hand, we know that $D_{3} / 4 v^{2}=\left(\lambda-\lambda_{1}\right)\left(\lambda-\lambda_{2}\right)$ with $\lambda_{1}=$ $\left(A+v^{2} B-D^{1 / 2}\right) / 2 v^{2}$ and $\lambda_{2}=\left(A+v^{2} B+D^{1 / 2}\right) / 2 v^{2}$, where $D=Z^{2}-v^{2} M^{2}$ is the discriminant of $D_{3} / 4$. Therefore the last inequality in (3.46) holds if

$$
\sup _{\xi} \lambda_{1}<\lambda<\inf _{\xi} \lambda_{2}
$$

Since $D \geqq \min \left\{D_{+}, D_{-}\right\}$and $D_{ \pm}=z_{ \pm}^{2}-v^{2} m_{ \pm}^{2} \geqq c_{0}^{2}$ with a positive constant $c_{0}$, the condition (3.51) is satisfied if

$$
\left(A_{\mp}+v^{2} B_{\mp}-c_{0}\right) / 2 v^{2}<\lambda<\left(A_{ \pm}+v^{2} B_{ \pm}+c_{0}\right) / 2 v^{2} \quad(\text { for } s \gtrless 0),
$$

where we also used $\left(3.41^{\prime}\right)$. The inequality $\left(A_{\mp}+v^{2} B_{\mp}-c_{0}\right) / 2 v^{2}<\left(A_{ \pm}+v^{2} B_{ \pm}+\right.$ $c_{0}$ ) $/ 2 v^{2}$ (for $s \gtrless 0$ ) is equivalent to

$$
v^{2}\left|\rho_{+}-\rho_{-}\right|+\left|z_{+}-z_{-}\right|<4 c_{0},
$$

which is satisfied automatically for suitably small $\left|\rho_{+}-\rho_{-}\right|$. Under the condition (3.53) we take $\lambda$ to be

$$
\lambda=\left(A_{+}+A_{-}\right) / 4 v^{2}+\left(B_{+}+B_{-}\right) / 4=\left(\rho_{+}+\rho_{-}\right) / 8+\left(z_{+}+z_{-}\right) / 8 v^{2} .
$$

Then (3.50) and (3.52) hold uniformly in $\left(\rho_{ \pm}, m_{ \pm}, z_{ \pm}\right)$. Thus condition (3.46) has been checked. This completes the proof of Lemma 3.5

For the derivative $\Phi_{\xi}$, we have the following

Lemma 3.6. There is a positive constant $C$ such that for $t \in[0, T]$, 


$$
\begin{aligned}
& \left\|\Phi_{\xi}(t)\right\|^{2}+\int_{0}^{t}\left\|\Phi_{\xi}(\tau)\right\|^{2} d \tau-C\left\{\left\|\left(\Psi_{\xi}, \Psi_{t}\right)(t)\right\|^{2}+\int_{0}^{t}\left\|\left(\Psi_{\xi}, \Psi_{t}\right)(\tau)\right\|^{2} d \tau\right\} \\
& \leqq C\left\{\left\|\Phi_{0, \xi}\right\|^{2}+\left\|w_{0}\right\|^{2}+\int_{0}^{t} \int\left|\Phi_{\xi}\right||\Gamma| d \xi d \tau\right\}
\end{aligned}
$$

Proof. We calculate the equality

$$
\left(\Psi_{t}-s \Psi_{\xi}+v^{2} \Phi_{\xi}\right) \cdot \partial_{\xi} L_{1}+\Phi_{\xi} \cdot L_{2}=-\Phi_{\xi} \cdot \Gamma
$$

The left-hand side of (3.56) is reduced to

$$
\left\{\frac{v^{2}}{2} \Phi_{\xi}^{2}+\Phi_{\xi}\left(\Psi_{t}-s \Psi_{\xi}\right)-\frac{1}{2} \Psi_{\xi}^{2}\right\}_{t}+A \Phi_{\xi}^{2}+\Phi_{\xi}\left\{(M-s B) \Psi_{\xi}+B \Psi_{t}\right\}+\{\cdots\}_{\xi} .
$$

Therefore, integrating (3.56) over $[0, t] \times \mathbb{R}$, we get the desired estimate (3.55). This completes the proof.

We proceed to estimate the higher derivatives.

Lemma 3.7. There is a positive constant $C$ such that for $t \in[0, T]$,

$$
\begin{aligned}
& \left\|\Phi_{\xi}(t)\right\|^{2}+\left\|\Psi_{\xi}(t)\right\|_{1}^{2}+\left\|\Psi_{t \xi}(t)\right\|^{2}+\int_{0}^{t}\left\|\left(\Psi_{\xi \xi}, \Psi_{t \xi}\right)(\tau)\right\|^{2} d \tau-C \int_{0}^{t}\left\|\left(\Phi_{\xi}, \Psi_{\xi}, \Psi_{t}\right)(\tau)\right\|^{2} d \tau \\
& \leqq C\left\{\left\|\Phi_{0, \xi}\right\|^{2}+\left\|\Psi_{0, \xi}\right\|_{1}^{2}+\left\|w_{0, \xi}\right\|^{2}+\int_{0}^{t} \int\left(\left|\Psi_{\xi}\right|+\left|\Psi_{\xi \xi}, \Psi_{t \xi}\right|\right)\left|\Gamma_{\xi}\right| d \xi d \tau\right\}, \\
& \left\|\Phi_{\xi \xi}(t)\right\|^{2}+\int_{0}^{t}\left\|\Phi_{\xi \xi}(\tau)\right\|^{2} d \tau-C\left\{\left\|\left(\Psi_{\xi \xi}, \Psi_{t \xi}\right)(t)\right\|^{2}+\int_{0}^{t}\left\|\Phi_{\xi}(\tau)\right\|^{2}+\left\|\left(\Psi_{\xi}, \Psi_{t}\right)(\tau)\right\|_{1}^{2} d \tau\right\} \\
& \leqq C\left\{\left\|\Phi_{0, \xi \xi}\right\|^{2}+\left\|w_{0, \xi}\right\|^{2}+\int_{0}^{t} \int\left|\Phi_{\xi \xi}\right|\left|\Gamma_{\xi}\right| d \xi d \tau\right\} .
\end{aligned}
$$

Proof. Since $\partial_{\xi} L_{1}(\Phi, \Psi)=L_{1}\left(\Phi_{\xi}, \Psi_{\xi}\right) \quad$ and $\quad \partial_{\xi} L_{2}(\Phi, \Psi)=L_{2}\left(\Phi_{\xi}, \Psi_{\xi}\right)+A_{\xi} \Phi_{\xi}+$ $(M-s B)_{\xi} \Psi_{\xi}+B_{\xi} \Psi_{t}$, we can calculate the equalities

$$
\begin{gathered}
-\partial_{\xi} \Psi_{\xi} \cdot \partial_{\xi} L_{1}+A^{-1} \partial_{\xi}\left(\Psi_{t}-s \Psi_{\xi}\right) \cdot \partial_{\xi} L_{2}=-A^{-1} \partial_{\xi}\left(\Psi_{t}-s \Psi_{\xi}\right) \cdot \partial_{\xi} \Gamma, \\
\partial_{\xi} \Phi \cdot \partial_{\xi} L_{1}+A^{-1} \partial_{\xi} \Psi \cdot \partial_{\xi} L_{2}=-A^{-1} \partial_{\xi} \Psi \cdot \partial_{\xi} \Gamma
\end{gathered}
$$

in the same way as in Lemma 3.5. Add (3.59) to $\lambda$ times (3.60), where $\lambda$ is the constant given in (3.54). Integrating the resulting equality over $[0, t] \times \mathbb{R}$, we get the desired estimate (3.57) as a counterpart of (3.40).

Next we calculate the equality

$$
\partial_{\xi}\left(\Psi_{t}-{ }_{s} \Psi_{\xi}+v^{2} \Phi_{\xi}\right) \cdot \partial_{\xi}^{2} L_{1}+\partial_{\xi} \Phi_{\xi} \cdot \partial_{\xi} L_{2}=-\partial_{\xi} \Phi_{\xi} \cdot \partial_{\xi} \Gamma
$$

in the same way as in Lemma 3.6. Integrating it over $[0, t] \times \mathbb{R}$, we get the estimate (3.58) as a counterpart of (3.55). This completes the proof of Lemma 3.7.

Combining successively the estimates (3.40), (3.55), (3.57) and (3.58), we have

$$
\begin{aligned}
N(t)^{2}+\int_{0}^{t}\left\|\left(\Phi_{\xi}, \Psi_{\xi}, \Psi_{t}\right)(\tau)\right\|_{1}^{2} d \tau \leqq & C\left\{N_{0}^{2}+\int_{0}^{t} \int\left|\Psi, \Phi_{\xi}, \Psi_{\xi}, \Psi_{t}\right||\Gamma|\right. \\
& \left.+\left|\Psi_{\xi}, \Phi_{\xi \xi}, \Psi_{\xi \xi}, \Psi_{t \xi}\right|\left|\Gamma_{\xi}\right| d \xi d \tau\right\}
\end{aligned}
$$


for $t \in[0, T]$, where $\left|\rho_{+}-\rho_{-}\right| \leqq \delta$ is assumed. Since

$$
|\Gamma|=0\left(\left|\Phi_{\xi}, \Psi_{\xi}, \Psi_{t}\right|^{2}\right) \text { for }\left|\Phi_{\xi}, \Psi_{\xi}, \Psi_{t}\right| \rightarrow 0,
$$

the integral on the right-hand side of (3.61) is majorized by

$$
C N(t) \int_{0}^{t}\left\|\left(\Phi_{\xi}, \Psi_{\xi}, \Psi_{t}\right)(\tau)\right\|_{1}^{2} d \tau
$$

with some constant $C$. Substituting it into (3.61), we reach the final estimate

$$
N(t)^{2}+(1-C N(t)) \int_{0}^{t}\left\|\left(\Phi_{\xi}, \Psi_{\xi}, \Psi_{t}\right)(\tau)\right\|_{1}^{2} d \tau \leqq C N_{0}^{2}
$$

for $t \in[0, T]$, where $C$ is a constant. Therefore, assuming $N(T) \leqq 1 / 2 C$, we obtain the desired estimate (3.39). Thus the proof of Proposition 3.4 is completed.

\section{References}

1. Caflisch, R. E.: Navier-Stokes and Boltzmann shock profiles for a model of gas dynamics. Commun Pure Appl. Math. 32, 521-554 (1979)

2. Gilbarg, D.: The existence and limit behavior of the one-dimensional shock layer. Am. J. Math. 73, 256-274 (1951)

3. Goodman, J.: Nonlinear asymptotic stability of viscous shock profiles for conservation laws (to appear)

4. Il'in A. M. Oleinik, O. A.: Asymptotic behavior of the solutions of the Cauchy problem for certain quasilinear equations for large time (Russian). Mat. Shornik 51, 191-216 (1960)

5. Jones, C.: Stability of the traveling wave solution of the FitzHugh-Nagumo system (to appear)

6. Matsumura, A., Nishihara, K.: On the stability of traveling wave solutions of a one-dimensional model system for compressible viscous gas, to appear in Jpn. J. Appl. Math

7. Nishida, T., Smoller, J. A.: Solutions in the large for some nonlinear hyperbolic conservation laws. Commun. Pure Appl. Math. 26, 183-200 (1983)

8. Nishihara, K.: A note on the stability of traveling wave solutions of the Burgers' equation, to appear in Jpn. J. Appl. Math

9. Vol'pert, A. I., Hudjaev, S. I.: On the Cauchy problem for composite systems of nonlinear differential equations. Math. USSR Sbornik 16, 517-544 (1972)

10. Kawashima, S., Nishida, T.: Global solutions to the initial value problem for the equations of onedimensional motion of viscous polytropic gases. J. Math. Kyoto Univ. 21, 825-837 (1981)

11. Matsumura, A., Nishida, T.: The initial value problem for the equations of motion of viscous and heat-conductive gases. J. Math. Kyoto Univ. 20, 67-104 (1980)

Communicated by L. Nirenberg

Received September 7, 1984; in revised form January 25, 1985 
